\title{
Some bijective correspondences involving domino tableaux
}

\author{
Marc A. A. van Leeuwen \\ Université de Poitiers, Département de Mathématiques, \\ UFR Sciences SP2MI, Téléport 2, BP 30179, 86962 Futuroscope Chasseneuil Cedex, France \\ maavl@mathlabo.univ-poitiers.fr \\ URL: http://wwwmathlabo.univ-poitiers.fr/〜maavl/
}

Submitted: April 13, 2000; Accepted: July 11, 2000.

\begin{abstract}
We define a number of new combinatorial operations on skew semistandard domino tableaux that complement constructions defined by $\mathrm{C}$. Carré and B. Leclerc, and clarify the link with ordinary skew semistandard tableaux and the Littlewood-Richardson rule. These operations are: (1) a bijection between semistandard domino tableaux and certain pairs of ordinary tableaux of the same weight that together fill the same shape, and which determine the "plactic class" of the domino tableau; (2) a weight preserving reversible transformation of domino tableaux into ordinary tableaux of a related shape (the correspondence involves 2-quotients) mapping the subset of Yamanouchi domino tableaux onto that of the Littlewood-Richardson tableaux; (3) a correspondence between Yamanouchi domino tableaux of shape $\lambda$ and weight $\mu$ and Yamanouchi domino tableaux of shape $\mu^{\prime}$ and weight $\lambda$, where $\mu^{\prime}$ is $\mu$ scaled horizontally and vertically by a factor 2 . The essential properties of (1) and (2) are obtained by proving their commutation with the "coplactic" (or crystal) operations (which for domino tableaux were defined by Carré and Leclerc). Construction (2) allows algorithmic separation of the Littlewood-Richardson tableaux describing the decomposition of the tensor square of a general linear group representation into contributions to its symmetric and alternating parts.
\end{abstract}

Mathematics Subject Classification 2000: 05E10.

Keywords and Phrases: domino tableau, bijective proof.

\section{$\S 1$. Introduction.}

The oldest form of the Robinson-Schensted correspondence, given in [Rob], associates to any semistandard skew tableaux $T$ of shape $\lambda / \mu$ and weight $\alpha$, a pair $(L, P)$ consisting, for some partition $\nu$, of a LittlewoodRichardson tableau $L$ of shape $\lambda / \mu$ and weight $\nu$, and a semistandard Young tableau $P$ of shape $\nu$ and weight $\alpha$ (cf. [vLee5, (8)]). In [CaLe], an analogue for domino tableaux of this construction was given, associating to a domino tableau $D$ a pair consisting of a so-called Yamanouchi domino tableau $Y$, and a semistandard Young tableau $P$. As a result, a decomposition rule for products of Schur functions similar to the Littlewood-Richardson rule is derived, counting Yamanouchi domino tableaux instead of Littlewood-Richardson tableaux; it has the additional advantage that for the square of a Schur function, its decomposition into its (representation theoretic) symmetric and alternating parts can be read off. 
In this paper we define three bijective constructions complementing these results. The first associates to a semistandard domino tableau a pair of ordinary semistandard tableaux called a self-switching tableau pair. This provides a direct description of the association $D \mapsto P$, of which the original description in [CaLe], like that of $T \mapsto P$ in [Rob], is quite indirect, and not even obviously well defined. The rôle of this first construction is therefore analogous to that of jeu de taquin, which describes the correspondence $T \mapsto P$. Our second construction is a weight preserving transformation of domino tableaux of shape $\lambda / \mu$ into ordinary tableaux of a shape determined by the 2-quotients of $\lambda$ and $\mu$, which maps Yamanouchi domino tableaux to Littlewood-Richardson tableaux; it relates the Littlewood-Richardson rule to its counterpart using domino tableaux. Our third construction defines a bijection between certain sets of Yamanouchi domino tableaux, which exhibits the identity of two different combinatorial expressions for the scalar product $\left\langle s_{\lambda}, \psi^{2}\left(s_{\mu}\right)\right\rangle$, given respectively in Corollary 4.3 and Theorem 5.3 of [CaLe].

To define these bijections, we apply algorithmic constructions defined elsewhere, notably in [vLee3] and [vLee5]. In addition, a central rôle is played by coplactic operations (cf. [vLee5, §3]), operating on domino tableaux; an implicit definition of these operations is contained in the algorithm [CaLe, 7.1].

This paper is organised as follows. In $\S 2$ we define our main bijective correspondences, and state (without proof) their fundamental properties. In $\S 3$ we give the definition of coplactic on domino tableaux used in this paper, which is in terms of so-called augmented domino tableaux. In $\S 4$ we relate these coplactic operations to those on ordinary tableaux; in particular the fundamental property of our first bijective correspondence is established by proving commutation with coplactic operations. In $\S 5$ we do the same for the other correspondences, which involve moving chains in domino tableaux.

The current paper depends strongly on notions developed in [vLee3] (of which we only use the special case $r=2$ of dominoes), and in [vLee5]; we shall now recall, and in some cases extend, their notations. The set of partition is denoted by $\mathcal{P}$, and it subset of 2-cores by $\mathcal{C}_{2}$. The parameters $d^{2}(\delta(\gamma))$ of a 2-core $\gamma$ always have the form $(c,-c)$ for $c \in \mathbf{Z}$, so we shall denote the 2-core so parametrised by $\gamma_{c}$; explicitly, $\gamma_{c}=(k, k-1, \ldots, 1)$, where $k=-2 c$ if $c \leq 0$ and $k=2 c-1$ if $c>0$. We define skew standard tableaux as sequences of partitions (saturated chains in the Young lattice); unlike in [vLee3] we do not specify a set of entries (an initial subset of $\mathbf{N}$ is always assumed). We define standard domino tableaux similarly, using the 2-rim hook lattice $\left(\mathcal{P}, \leq_{2}\right)$ instead of the Young lattice. Semistandard (domino) tableaux are determined by their standardisation and their weight. We write $\operatorname{SST}(\lambda / \mu)$ for the set of all semistandard tableaux of shape $\lambda / \mu$, and $\operatorname{SSDT}(\lambda / \mu)$ for the analogous set of semistandard domino tableaux; when restricting to entries $<n$, we write $\operatorname{SST}(\lambda / \mu, n)$ and $\operatorname{SSDT}(\lambda / \mu, n)$. We denote the set of dominoes of $D \in \operatorname{SSDT}(\lambda / \mu)$ by $\operatorname{Dom}(D)$; the set of squares of $T \in \operatorname{SSDT}(\lambda / \mu)$ is just the Young diagram $Y(\lambda / \mu)$, independently of $T$. If moreover $s \in Y(\lambda / \mu)$ and $x \in \operatorname{Dom}(D)$, we write $T(s)$ respectively $D(x)$ for the entries of the square $s$ and domino $x$, and $\operatorname{pos}(s), \operatorname{pos}(x)$ for their respective positions (i.e., maximal index of a diagonal meeting the square/domino). Furthermore we shall use the spin $\operatorname{Spin}(D)$ of $D$ (half the number of vertical dominoes in $\operatorname{Dom}(D))$, and the 2 -sign $\varepsilon_{2}(\lambda / \mu)=(-1)^{2 \operatorname{Spin}(D)}$ of its shape.

The affine permutation group $\tilde{\mathbf{S}}_{2}$ is freely generated by two involutions $s_{0}, s_{1}$; each of them defines a structure of chains on the set of dominoes of any domino tableau $D$. Two actions of $\tilde{\mathbf{S}}_{2}$ are defined on semistandard domino tableaux: in the first, denoted by $\sigma(D)$, the generator $s_{i}$ moves all chains of $D$ for $s_{i}$ that are not forbidden (cf. [vLee3, proposition 4.3.1]); in the second, denoted by $\sigma \circ D$, it moves only the subset of open chains (it follows that $\sigma(D)$ and $\sigma \circ D$ have the same shape). Tableau switching, which is an involution on pairs $(S, T)$ of skew semistandard tableaux whose shape fit together (i.e., $S \in \operatorname{SST}(\mu / \nu)$, $T \in \operatorname{SST}(\lambda / \mu)$, for some $\lambda, \mu, \nu \in \mathcal{P})$, is denoted by $X(S, T)$, and the relations generated by inward respectively outward jeu de taquin slides by $T \triangleright T^{\prime}$ and $S \triangleleft S^{\prime}$ (when $X(S, T)=\left(T^{\prime}, S^{\prime}\right)$, these relations hold; indeed they are equivalent to having this identity for some $S, S^{\prime}$, respectively for some $\left.T, T^{\prime}\right)$.

\section{Acknowledgements.}

I wish to thank Mark Shimozono for calling my attention to coplactic operations and their relation to operations on pictures. I also wish to thank Bernard Leclerc for then many stimulating discussions concerning the constructions of [CaLe], and in particular their relation with coplactic graphs. 


\section{$\S 2$. Various bijective correspondences for domino tableaux.}

In this section we formulate three results about domino tableaux that complement those found in [CaLe]. We introduce the algorithmic constructions involved, but postpone proofs of most of their properties. We use several concepts and constructions that were introduced in [CaLe], most notably the concept of Yamanouchi domino tableaux (a subclass of the semistandard domino tableaux analogous to that of Littlewood-Richardson tableaux for ordinary semistandard tableaux) and the bijection of [CaLe, Theorem 7.3]. Formally our Yamanouchi domino tableaux differ from those of [CaLe] in that their entries start from 0 (to remain consistent with our other definitions), but this just amounts to a trivial renumbering.

\subsection{Projection from domino tableaux to Young tableaux.}

In this subsection we define a weight preserving map from semistandard domino tableaux to Young tableaux, which will coincide with the projection onto the second factor after applying the bijection of [CaLe, Theorem 7.3].

As mentioned in the introduction, jeu de taquin can be defined in terms of tableau switching. On its turn tableau switching is described using so-called tableau switching families of partitions $\left(\lambda^{[i, j]}\right)_{i \in I, j \in J}$, where $I=\{k, \ldots, l\}$ and $J=\{m, \ldots, n\}$ are of intervals of $\mathbf{Z}$. When $S, T, S^{\prime}, T^{\prime}$ are skew standard tableaux with $X(S, T)=\left(T^{\prime}, S^{\prime}\right)$, then there is such a family such that $S$ and $T$ are respectively read off along the left $(j=m)$ and bottom $(i=l)$ edges of the family, while $T^{\prime}$ and $S^{\prime}$ are respectively read off along its top $(i=k)$ and right $(j=n)$ edges. The conditions for a tableau switching family are such that either $(S, T)$ or $\left(T^{\prime}, S^{\prime}\right)$ determine the entire family. If it happens that $(S, T)=X(S, T)$, we call $(S, T)$ a self-switching standard tableau pair; the associated tableau switching family with $I=J$ is symmetric: $\lambda^{[i, j]}=\lambda^{[j, i]}$ for all $i, j \in I$. By the definition of a tableau switching family, such symmetry arises if and only if the diagonal sub-family $D=\left(\lambda^{[i, i]}\right)_{i \in I}$ is a skew standard domino tableau, and each such domino tableau $D$ corresponds to a unique symmetric tableau switching family (the argument is the same as given in [vLee1, §2.3]). In particular, $D$ determines $(S, T)$, and $\pi_{0}: D \mapsto(S, T)$ defines a shape preserving bijection $\pi_{0}$ from skew standard domino tableaux to self-switching standard tableau pairs, where the shape of $(S, T)$ is taken to be that of the tableau $S \mid T$ obtained by joining the two skew tableaux.

As is the case with tableau switching, the bijection $\pi_{0}$ can be extended to the case of semistandard tableaux; the resulting operation will be denoted by $\pi_{1}$. A pair $(U, V)$ of semistandard tableaux satisfying $X(U, V)=(U, V)$ is called self-switching; this means that their standardisations form a self-switching standard tableau pair, and that wt $U=$ wt $V$ (which we call the weight of the pair). For a semistandard domino tableau $D$ with standardisation $D_{0}$ and weight $\alpha$, we shall define $\pi_{1}(D)$ as the pair $(U, V)$ of semistandard tableaux with weight $\alpha$ and standardisations $(S, T)=\pi_{0}\left(D_{0}\right)$; to justify this, we must verify that $S$ and $T$ are compatible with $\alpha$. Let $d=\lambda^{[i, i]}-\lambda^{[i-1, i-1]}$ and $d^{\prime}=\lambda^{[i+1, i+1]}-\lambda^{[i, i]}$ be successive dominoes of $D$ with equal entries, so that $\operatorname{pos}(d)<\operatorname{pos}\left(d^{\prime}\right)$ by the definition of semistandard domino tableaux. This is easily seen to imply that the squares $s=\lambda^{[i, i]}-\lambda^{[i, i-1]}$ and $s^{\prime}=\lambda^{[i, i+1]}-\lambda^{[i, i]}$ satisfy $\operatorname{pos}(s)<\operatorname{pos}\left(s^{\prime}\right)$; therefore the skew standard tableau $K$ given by row $i$ of the tableau switching family satisfies compatibility with $\alpha$ at the pair of entries under consideration. This compatibility is preserved by jeu de taquin, so since $S \triangleleft K \triangleleft T$, it holds for $S$ and $T$ as well. We have proved:

2.1.1. Proposition. The correspondence $\pi_{1}$ defines a shape and weight preserving bijection from semistandard domino tableaux to self-switching tableau pairs.

As was done for tableau switching in [vLee $5, \S 2.2]$, we may deduce a description of the computation of $\pi_{1}(D)$ in terms of sliding entries of two different colours within the skew diagram. By symmetry, we need only determine the upper triangular half of the tableau switching family. We label each vertical difference $\lambda^{[i+1, j]}-\lambda^{[i, j]}$ by a red entry, and each horizontal difference by a blue one; for each colour, the multiset of entries is that of $D$. At each stage in the sliding process one has a shuffle of the weakly increasing sequences of red and blue numbers which, by concatenation of the associated vertical and horizontal steps, determines a lattice path through the tableau switching family. Each number in the shuffle corresponds to an entry in the diagram; among equal numbers of the same colour, the correspondence preserves left to right order. Whenever a red and blue number are transposed in the shuffle, the corresponding 
entries in the diagram are interchanged if and only if they are in adjacent squares. Initially each domino of $D$ is filled with a blue copy of its entry in its inward square and a red copy in its outward square, corresponding to the shuffle in which each red number immediately follows the same blue number. This shuffle is repeatedly modified until all blue numbers precede all red ones, at which point the blue and red entries define the two component tableaux of $\pi_{1}(D)$. For example, for the computation of

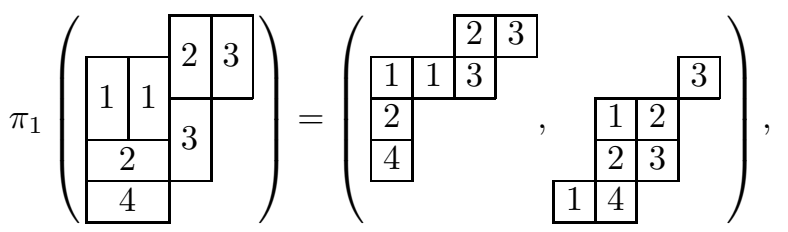

some intermediate stages, with their shuffles, are (with bold face representing blue, and italics red):

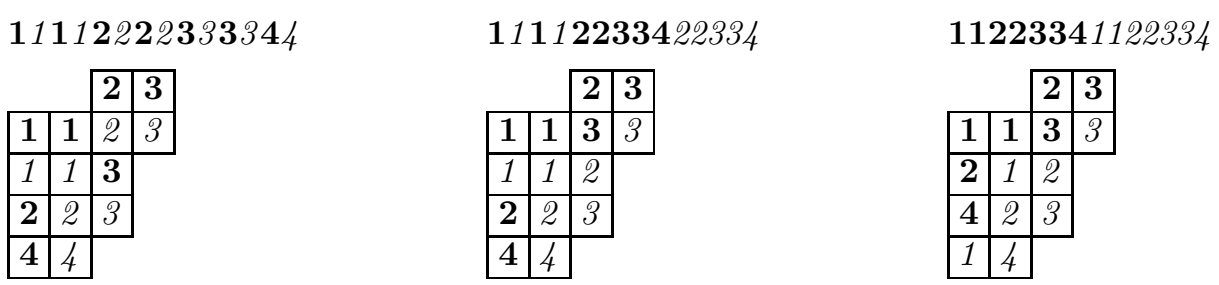

Reordering the shuffle from right to left, as in the example, amounts to performing inward jeu de taquin slides on the blue entries, into the squares indicated by the red entries, in decreasing order. Reordering from the left would amount to performing outward slides on the red entries into the squares of the blue ones, in increasing order. Since for $(U, V)=\pi_{1}(D)$ we have $U \triangleleft V$ by construction, we can define:

2.1.2. Definition. A weight preserving map $\pi$ from the set of semistandard skew domino tableaux to the set of semistandard Young tableaux is defined by the condition $\pi(D) \triangleleft U \triangleleft V$, where $(U, V)=\pi_{1}(D)$.

2.1.3. Theorem. For any domino tableau $D$, the second component of the pair associated to it by the bijection of [CaLe, Theorem 7.3] is equal to $\pi(D)$. In particular, if wt $D=\lambda \in \mathcal{P}$, then $D$ is a Yamanouchi domino tableau if and only if $\pi(D)=\mathbf{1}_{\lambda}$, the unique Young tableau with shape and weight $\lambda$.

For instance, for the domino tableau $D$ of $(1)$ we have $\pi(D)=\frac{\frac{11}{2}^{2 / 3}}{\frac{3}{4}}$, in agreement with the Young tableau computed in [CaLe, 7.2, example 2]. The method by which the Young tableau is obtained is entirely different however, and the proof of theorem 2.1.3 will be a rather indirect one.

\subsection{Yamanouchi domino tableaux and Littlewood-Richardson tableaux.}

In [CaLe, Corollary 4.4] Littlewood-Richardson coefficients are expressed as the cardinalities of certain sets of Yamanouchi domino tableaux. We shall exhibit an algorithmic bijection from the corresponding sets of Littlewood-Richardson tableaux to these sets of domino tableaux. In particular this defines a partitioning of the set of Littlewood-Richardson tableaux describing the square of a Schur function, into contributions to the symmetric and alternating part of the square, by means of the spins of the associated Yamanouchi domino tableaux. These spins cannot be predicted without performing the algorithm, which may explain why earlier attempts to describe such a partitioning by combinatorial means have failed.

We first give a general expression of the multiplication of skew Schur functions in terms of Yamanouchi domino tableaux (only a special case is stated explicitly in [CaLe]), after introducing some notation for skew shapes and tableaux characterised by 2-quotients and 2-cores.

2.2.1. Definition. For $i=0,1$ let $\lambda^{(i)} / \mu^{(i)}$ be a skew shape, $T_{i} \in \operatorname{SST}\left(\lambda^{(i)} / \mu^{(i)}\right)$, and let $\gamma \in \mathcal{C}_{2}$.

(1) $\mathrm{cq}_{2}\left(\gamma, \lambda^{(0)}, \lambda^{(1)}\right)$ is the unique partition with 2-core $\gamma$ and 2-quotient $\left(\lambda^{(0)}, \lambda^{(1)}\right)$;

(2) $\operatorname{cq}_{2}\left(\gamma, \lambda^{(0)} / \mu^{(0)}, \lambda^{(1)} / \mu^{(1)}\right)=\mathrm{cq}_{2}\left(\gamma, \lambda^{(0)}, \lambda^{(1)}\right) / \mathrm{cq}_{2}\left(\gamma, \mu^{(0)}, \mu^{(1)}\right)$;

(3) $\operatorname{cq}_{2}\left(\gamma, T_{0}, T_{1}\right)$ is the semistandard domino tableau of $\operatorname{shape} \operatorname{cq}_{2}\left(\gamma, \lambda^{(0)} / \mu^{(0)}, \lambda^{(1)} / \mu^{(1)}\right)$ corresponding to $\left(T_{0}, T_{1}\right)$ under the bijection of [vLee3, proposition 3.2.2]. 
2.2.2. Theorem [Carré \& Leclerc]. Let $\chi, \chi^{\prime}$ be skew shapes and $\gamma \in \mathcal{C}_{2}$; then

$$
s_{\chi} \cdot s_{\chi^{\prime}}=\sum_{\nu \in \mathcal{P}} \# \operatorname{Yam}_{2}\left(\mathrm{cq}_{2}\left(\gamma, \chi, \chi^{\prime}\right), \nu\right) s_{\nu}
$$

where $\operatorname{Yam}_{2}(\psi, \nu)$ denotes the set of all Yamanouchi domino tableaux of shape $\psi$ and weight $\nu$.

Proof. By [vLee3, corollary 3.2.3] we have

$$
\sum_{D \in \operatorname{SSDT}\left(\mathrm{cq}_{2}\left(\gamma, \chi, \chi^{\prime}\right), n\right)} x^{\mathrm{wt}(D)}=s_{\chi}(n) \cdot s_{\chi^{\prime}}(n),
$$

while by [CaLe, Theorem 7.3],

$$
\sum_{D \in \operatorname{SSDT}(\psi, n)} x^{\mathrm{wt}(D)}=\sum_{\nu \in \mathcal{P}}\left(\# \operatorname{Yam}_{2}(\psi, \nu) \sum_{T \in \operatorname{SST}(\nu, n)} x^{\mathrm{wt}(T)}\right)=\sum_{\nu \in \mathcal{P}} \# \operatorname{Yam}_{2}(\psi, \nu) s_{\nu}(n) .
$$

It follows as a special case that the Littlewood-Richardson coefficient $c_{\lambda, \lambda^{\prime}}^{\nu}$ is equal to \# $\operatorname{Yam}_{2}(\psi, \nu)$, where $\psi$ is the skew shape $\mathrm{cq}_{2}\left(\gamma, \lambda / 0, \lambda^{\prime} / 0\right)=\mathrm{cq}_{2}\left(\gamma, \lambda, \lambda^{\prime}\right) / \gamma$, for an arbitrary 2 -core $\gamma$. This coefficient is traditionally described as the cardinality of one of several sets of Littlewood-Richardson tableaux (see for instance [vLee2, 2.6]); we choose the set $\operatorname{LR}\left(\lambda * \lambda^{\prime}, \nu\right)$ of such tableaux of the shape $\lambda * \lambda^{\prime}$ and weight $\nu$, where $\lambda * \lambda^{\prime}$ is the skew shape obtained by attaching the diagram of $\lambda$ to the left and below that of $\lambda^{\prime}$. The general case of the theorem then has a similar interpretation: the cardinality of $\operatorname{Yam}_{2}\left(\mathrm{cq}_{2}\left(\gamma, \chi, \chi^{\prime}\right), \nu\right)$ is equal to that of $\operatorname{LR}\left(\chi * \chi^{\prime}, \nu\right) . \dagger$ We shall give a bijective proof of this identity: for any 2-core $\gamma$ and any skew shapes $\chi, \chi^{\prime}$ we shall construct a weight preserving bijection $\operatorname{LR}\left(\chi * \chi^{\prime}\right) \rightarrow \operatorname{Yam}_{2}\left(\mathrm{cq}_{2}\left(\gamma, \chi, \chi^{\prime}\right)\right)$. In fact, the nature of the construction is such that it simultaneously defines a weight preserving bijection $\operatorname{LR}\left(\chi * \chi^{\prime}\right) \rightarrow \operatorname{Yam}_{2}\left(\mathrm{cq}_{2}\left(\gamma, \chi^{\prime}, \chi\right)\right)$ as well; this is remarkable since there is no obvious bijection between $\operatorname{LR}\left(\chi * \chi^{\prime}\right)$ and $\operatorname{LR}\left(\chi^{\prime} * \chi\right)$. These bijections will be obtained by restriction of appropriate weight preserving bijections from $\operatorname{SST}\left(\chi * \chi^{\prime}\right)$ to $\operatorname{SSDT}\left(\mathrm{cq}_{2}\left(\gamma, \chi, \chi^{\prime}\right)\right)$ and $\operatorname{SSDT}\left(\mathrm{cq}_{2}\left(\gamma, \chi^{\prime}, \chi\right)\right)$; to this end we must find such bijections that map Littlewood-Richardson tableaux precisely to Yamanouchi domino tableaux.

Without the final condition, such weight preserving bijections are already provided by the maps $\Sigma_{c}: T_{0} * T_{1} \mapsto \mathrm{cq}_{2}\left(\gamma_{c}, T_{0}, T_{1}\right)$ and $\Sigma_{c}^{\prime}: T_{0} * T_{1} \mapsto \mathrm{cq}_{2}\left(\gamma_{c}, T_{1}, T_{0}\right)$, for $c \in \mathbf{Z}$; these do not however in general map Littlewood-Richardson tableaux to Yamanouchi domino tableaux. This can be understood from the definitions of such tableaux, which require the word formed by reading the entries of the tableau in a particular order to be a lattice permutation: for Littlewood-Richardson tableaux this can be any valid reading order as described in [vLee5, §1.5], while for Yamanouchi domino tableaux this is the reverse of the column reading order of [CaLe] (a Yamanouchi word is a reverse lattice permutation). Each entry of $\Sigma_{c}\left(T_{0} * T_{1}\right)$ or $\Sigma_{c}^{\prime}\left(T_{0} * T_{1}\right)$ has a matching entry in $T_{0} * T_{1}$, but the reading order used in the domino tableau does not always correspond to a valid reading order in $T_{0} * T_{1}$. This even fails for the components $T_{0}$ and $T_{1}$ individually, but more importantly, their entries are interleaved in the reading of the domino tableau, while all entries of $T_{1}$ precede those of $T_{0}$ first factor in any valid reading of $T_{0} * T_{1}$.

However, if $|c|$ is sufficiently large, the situation is different. According to [vLee3, proposition 3.1.2], a domino $d$ in $\mathrm{cq}_{2}\left(\gamma_{c}, U, V\right)$ corresponding to a square $s$ of $U$ satisfies $\operatorname{pos}(d)=2(\operatorname{pos}(s)+c)$, while it satisfies $\operatorname{pos}(d)=2(\operatorname{pos}(s)-c)+1$ if it corresponds to a square $s$ of $V$. Therefore $\Sigma_{c}$ preserves the relative order of positions when $c \ll 0$, and $\Sigma_{c}^{\prime}$ does so when $c \gg 0$. Here is a concrete exemple to illustrate what

$\dagger$ Strictly speaking, we did not unambiguously define the shape $\chi * \chi^{\prime}$, and if we did, we would not be able to reconstruct $\chi$ and $\chi^{\prime}$ uniquely from it in all cases. Formally we define tableaux $T_{0} * T_{1} \in \operatorname{SST}\left(\chi * \chi^{\prime}\right)$ simply as pairs $\left(T_{0}, T_{1}\right) \in \operatorname{SST}(\chi) \times \operatorname{SST}\left(\chi^{\prime}\right)$, but with the convention that, for the purpose of reading orders (as in defining $\left.\operatorname{LR}\left(\chi * \chi^{\prime}, \nu\right)\right)$, all entries of $T_{1}$ are considered to lie above and to the right of those of $T_{0}$. 
happens: we display $T_{0} * T_{1}$, followed by $\Sigma_{3}^{\prime}\left(T_{0} * T_{1}\right), \quad \Sigma_{-2}\left(T_{0} * T_{1}\right), \quad \Sigma_{2}^{\prime}\left(T_{0} * T_{1}\right)$, and $\Sigma_{-1}\left(T_{0} * T_{1}\right)$.
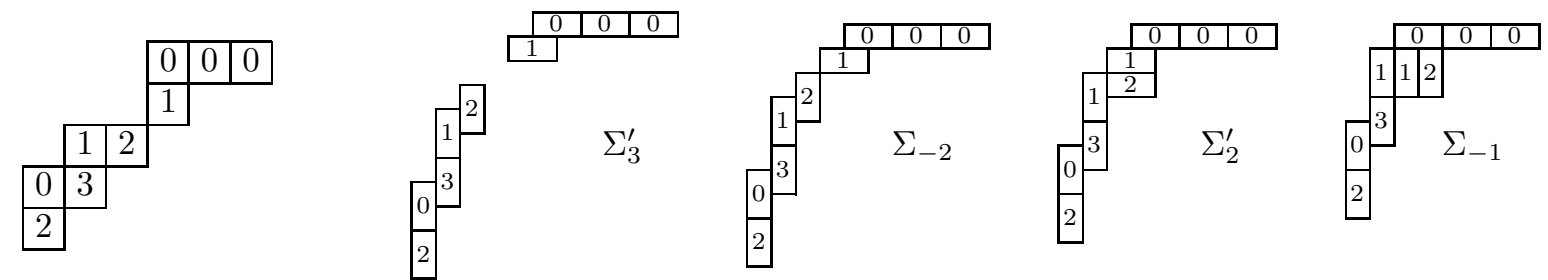

In the first two domino tableaux displayed, the dominoes corresponding to squares of $T_{0}$ and $T_{1}$ form disjoint subtableaux, the former consisting entirely of vertical dominoes and the latter of horizontal ones; in both components the correspondence between squares and dominoes is linear, and independent of the other component. Then the reading order in $T_{0} * T_{1}$ induced by the column reading order for the domino tableau is a valid one, so the fact that $T_{0} * T_{1}$ is a Littlewood-Richardson tableau implies that the indicated domino tableaux are Yamanouchi. For the third domino tableaux above most of these statements loose their validity, and the fourth one is in fact no longer a Yamanouchi domino tableau. The condition that characterises the simpler situation in the first two domino tableaux can be stated in terms of the dominoes $d$ and $d^{\prime}$ corresponding respectively to the top-right square $s$ of $T_{0}$ and the bottom-left square $t$ of $T_{1}$ : it is $\operatorname{pos}(d) \leq \operatorname{pos}\left(d^{\prime}\right)-3$ (so that the diagonal with index $\operatorname{pos}(d)+1$ separates the two components), which in view of the expression given above becomes $2 c \leq \operatorname{pos}(s)-\operatorname{pos}(t)-1$ in case of $\Sigma_{c}$, and $2 c \geq \operatorname{pos}(t)-\operatorname{pos}(s)+2$ for $\Sigma_{c}^{\prime}$. Indeed, in the example $\operatorname{pos}(s)-\operatorname{pos}(t)=-1-2=-3$, so these conditions are met for $c \leq-2$ respectively for $c \geq 3$. Concluding, our reasoning leads to the following definition and proposition.

2.2.3. Definition. Let $T_{0} \in \operatorname{SST}(\lambda / \mu)$ and $T_{1} \in \operatorname{SST}\left(\lambda^{\prime} / \mu^{\prime}\right)$; then $\Sigma_{c}\left(T_{0} * T_{1}\right)$ (respectively $\Sigma_{c}^{\prime}\left(T_{0} * T_{1}\right)$ ) is called a segregated tableau for $T_{0} * T_{1}$ when $2 c \leq-n+1$ (respectively when $2 c \geq n$ ), where $n=\lambda_{0}+\left(\lambda^{\prime}\right)_{0}^{\mathrm{t}}$.

2.2.4. Proposition. If a domino tableau $\Sigma_{c}\left(T_{0} * T_{1}\right)$ or $\Sigma_{c}^{\prime}\left(T_{0} * T_{1}\right)$ is segregated, then it is a Yamanouchi domino tableau if and only if $T_{0} * T_{1}$ is an Littlewood-Richardson tableau.

Our goal is now to extend the proposition by replacing $\Sigma_{c}\left(T_{0} * T_{1}\right)$ and $\Sigma_{c}^{\prime}\left(T_{0} * T_{1}\right)$, in case they are not segregated, by appropriate other domino tableaux of the same shape and weight. To this end observe that the collection of all domino tableaux $\Sigma_{c}\left(T_{0} * T_{1}\right)$ and $\Sigma_{c}^{\prime}\left(T_{0} * T_{1}\right)$ for $c \in \mathbf{Z}$ forms an orbit for the action $(\sigma, D) \mapsto \sigma(D)$ of the group $\tilde{\mathbf{S}}_{2}$ on semistandard domino tableaux. Indeed $s_{i}\left(\mathrm{cq}_{2}\left(\gamma, T_{0}, T_{1}\right)\right)=$ $\mathrm{cq}_{2}\left(s_{i}(\gamma), T_{1}, T_{0}\right)$ for $i=0,1$ by the description of [vLee3, proposition 4.3.2], while $s_{0}\left(\gamma_{c}\right)=\gamma_{1-c}$ and $s_{1}\left(\gamma_{c}\right)=\gamma_{-c}$; the orbit can be depicted as follows (omitting the arguments $T_{0} * T_{1}$ ):

$$
\cdots \Sigma_{2}^{\prime} \stackrel{s_{0}}{\longleftrightarrow} \Sigma_{-1} \stackrel{s_{1}}{\longleftrightarrow} \Sigma_{1}^{\prime} \stackrel{s_{0}}{\longleftrightarrow} \Sigma_{0} \stackrel{s_{1}}{\longleftrightarrow} \Sigma_{0}^{\prime} \stackrel{s_{0}}{\longleftrightarrow} \Sigma_{1} \stackrel{s_{1}}{\longleftrightarrow} \Sigma_{-1}^{\prime} \stackrel{s_{0}}{\longleftrightarrow} \Sigma_{2} \ldots
$$

If we go far enough to the left in this diagram, the domino tableaux $\Sigma_{c}$ and $\Sigma_{c}^{\prime}$ will be segregated. We shall choose such a segregated domino tableau $S$ in this orbit, and then replace the orbit by the orbit of $S$ for the other action $(\sigma, D) \mapsto \sigma \circ D$ of $\tilde{\mathbf{S}}_{2}$ (cf. [vLee3, proposition 4.5.2]). Recall that whereas in the former action application of $s_{i}$ amounts to moving all non-forbidden chains for $s_{i}$, this is limited in the latter action to moving the subset of open chains. Since it is easily seen that a segregated tableau contains only open chains (both for $s_{0}$ and for $s_{1}$ ), the part of the two orbits consisting of segregated tableaux will be identical, whence our construction is independent of the choice of $S$.

2.2.5. Definition. For any skew shapes $\chi, \chi^{\prime}$ and $c \in \mathbf{Z}$, maps $\Phi_{c}$ and $\Phi_{c}^{\prime}$ from $\operatorname{SST}\left(\chi * \chi^{\prime}\right)$ to respectively $\operatorname{SSDT}\left(\mathrm{cq}_{2}\left(\gamma_{3} \chi, \chi^{\prime}\right)\right)$ and $\operatorname{SSDT}\left(\mathrm{cq}_{2}\left(\gamma_{3} \chi^{\prime}, \chi\right)\right)$ are defined as follows. Let $S$ be a segregated domino tableau for $T_{0} * T_{1} \in \operatorname{SST}\left(\chi * \chi^{\prime}\right)$; then $\Phi_{c}\left(T_{0} * T_{1}\right)$ and $\Phi_{c}^{\prime}\left(T_{0} * T_{1}\right)$ are the unique elements in the orbit of $S$ for the action $(\sigma, D) \mapsto \sigma \circ D$, of respective shapes $\mathrm{cq}_{2}\left(\gamma, \chi, \chi^{\prime}\right)$ and $\mathrm{cq}_{2}\left(\gamma, \chi^{\prime}, \chi\right):$ if $\sigma, \sigma^{\prime} \in \tilde{\mathbf{S}}_{2}$ are such that $\sigma(S)=\Sigma_{c}\left(T_{0} * T_{1}\right)$ and $\sigma^{\prime}(S)=\Sigma_{c}^{\prime}\left(T_{0} * T_{1}\right)$, then $\Phi_{c}\left(T_{0} * T_{1}\right)=\sigma \circ S$ and $\Phi_{c}^{\prime}\left(T_{0} * T_{1}\right)=\sigma^{\prime} \circ S$.

As an example we compute $\Phi_{1}\left(T_{0} * T_{1}\right)$ for the tableau $T_{0} * T_{1}$ of the previous example. We start with the third domino tableau desplayed earlier, which is the first non-segregated one, and is part of the orbit for either of the actions; after this point there is divergence, and the action $(\sigma, D) \mapsto \sigma \circ D$ proceeds: 

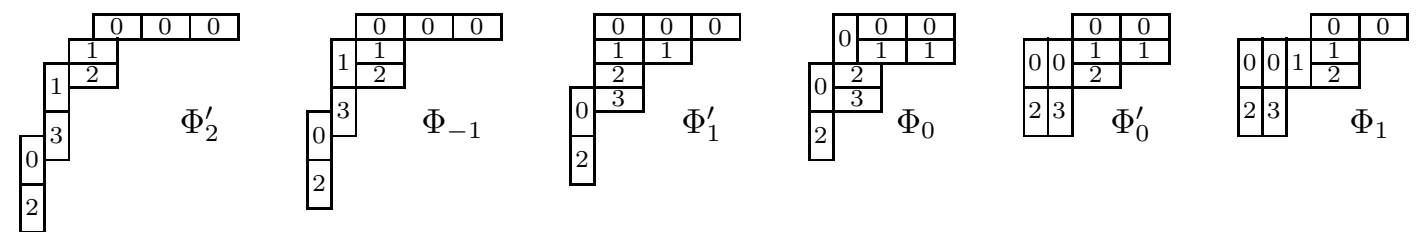

Note that these are all Yamanouchi domino tableaux. As we shall prove, this is no coincidence:

2.2.6. Theorem. By restriction, $\Phi_{c}$ and $\Phi_{c}^{\prime}$ define, for any $c \in \mathbf{Z}$, bijections from $\operatorname{LR}\left(\chi * \chi^{\prime}\right)$ respectively to $\operatorname{Yam}_{2}\left(\mathrm{cq}_{2}\left(\gamma_{c}, \chi, \chi^{\prime}\right)\right)$ and to $\operatorname{Yam}_{2}\left(\mathrm{cq}_{2}\left(\gamma_{c}, \chi^{\prime}, \chi\right)\right)$.

We shall in fact prove the stronger statement that moving any open chain is compatible with the bijection of [CaLe, Theorem 7.3]: on the first factor (the Yamanouchi domino tableau) the corresponding open chain is moved, while the second factor (the Young tableau) is unchanged.

Concerning definition 2.2.5, we note the following. While, as indicated in the example, it is clear where in the orbit each $\Phi_{c}\left(T_{0} * T_{1}\right)$ and $\Phi_{c}^{\prime}\left(T_{0} * T_{1}\right)$ are to be found, we have initially defined these tableaux as being uniquely determined within the orbit by their shape. When the shapes $\chi$ and $\chi^{\prime}$ of $T_{0}$ and $T_{1}$ are distinct, this is true because the action of $\tilde{\mathbf{S}}_{2}$ on the shapes in the orbit is free; on the other hand when $\chi=\chi^{\prime}$, each shape has a stabiliser of order 2 , since $\mathrm{cq}_{2}\left(\gamma_{0}, \chi, \chi\right)$ is stabilised by $s_{1}$. In the latter case however the tableau $\Phi_{0}\left(T_{0} * T_{1}\right)$ is also stabilised by $s_{1}$ (i.e., $\Phi_{0}\left(T_{0} * T_{1}\right)=\Phi_{0}^{\prime}\left(T_{0} * T_{1}\right)$ ), since the fact that the shape does not change implies that all chains are closed; then the whole orbit is symmetric and each tableau is unique for its shape. This also implies that $\Phi_{c}$ and $\Phi_{c}^{\prime}$ coincide for $\chi=\chi^{\prime}$.

Another point that can be observed is that the image of the map $\Phi_{c}$ for the shape $\chi * \chi^{\prime}$ coincides with the image of $\Phi_{c}^{\prime}$ for $\chi^{\prime} * \chi$. Therefore we may compose the former map with the inverse of the latter, so as to obtain a bijection $\operatorname{SST}\left(\chi * \chi^{\prime}\right) \rightarrow \operatorname{SST}\left(\chi^{\prime} * \chi\right)$ which, by theorem 2.2.6, restricts to a bijection $X_{\text {Dom }}: \operatorname{LR}\left(\chi * \chi^{\prime}\right) \rightarrow \operatorname{LR}\left(\chi^{\prime} * \chi\right)$; the following description shows that $X_{\mathrm{Dom}}\left(T_{0} * T_{1}\right)$ does not depend on $c$. One forms segregated tableau for $T_{0} * T_{1}$, and moves though the non-segregated part of its orbit for the action $(\sigma, D) \mapsto \sigma \circ D$, to the other (right) end, where the domino tableau becomes segregated again, but for some other tableau $T_{1}^{\prime} * T_{0}^{\prime}=X_{\text {Dom }}\left(T_{0} * T_{1}\right)$ of shape $\chi^{\prime} * \chi$. This bijection differs from the "traditional" bijection $X_{\mathrm{LR}}: \operatorname{LR}\left(\chi * \chi^{\prime}\right) \rightarrow \operatorname{LR}\left(\chi^{\prime} * \chi\right)$ that is described in detail in [vLee4], and can be characterised in the language of [vLee5] as tableau switching on companion tableaux. In fact the two bijections have rather different characteristics: in $X_{\text {Dom }}$ the shapes $\chi, \chi^{\prime}$ play a crucial rôle (e.g., $X_{\text {Dom }}$ is the identity for $\chi=\chi^{\prime}$, by the remarks above), whereas $X_{\mathrm{LR}}$ only uses some reading of the tableaux (a lattice permutation); also $X_{\mathrm{LR}}$ does not naturally extend to a bijection $\operatorname{SST}\left(\chi * \chi^{\prime}\right) \rightarrow \operatorname{SST}\left(\chi^{\prime} * \chi\right)$.

2.3. Matching two expressions for $\left\langle s_{\lambda}, \psi^{2}\left(s_{\mu}\right)\right\rangle$.

Our third construction establishes a bijection corresponding to the identity

$$
\varepsilon_{2}(\lambda) \# \operatorname{Yam}_{2}(\lambda, \mu)=\sum_{M \in \operatorname{Yam}_{2}\left(\mu^{\square}, \lambda\right)}(-1)^{|\mu|-\operatorname{Spin}(M)},
$$

where $\mu^{\square}=\mathrm{cq}_{2}(\emptyset, \mu, \mu)$ is the partition obtained from the Young diagram of $\mu$ by scaling up by a factor 2 both horizontally and vertically. Since $\operatorname{Spin}(D)$ denotes half the number of vertical dominoes in $D$, and $\varepsilon_{2}(\lambda)=\varepsilon_{2}(\lambda / \emptyset)=(-1)^{2} \operatorname{Spin}(D)$ for any $D \in \operatorname{SSDT}(\lambda)$, the Yamanouchi domino tableaux in the first member are counted with a fixed sign determined by the parity of their number of vertical dominoes, while those in the second member are counted with a varying sign, determined by the parity of half the number of horizontal dominoes (since $\varepsilon_{2}\left(\mu^{\square}\right)=1$, and these tableaux have $2|\mu|$ dominoes altogether).

We recall that both members of (2) are combinatorial expressions for the number $\left\langle s_{\lambda}, \psi^{2}\left(s_{\mu}\right)\right\rangle$, where $\psi^{2}$ is the plethysm operator that replaces each monomial $x^{\alpha}$ by $x^{2 \alpha}$. We review briefly the derivation of this identity, as it is spread across many sections of [CaLe], and since similar arguments will be used in our bijective proof. Denoting by $\phi^{2}$ the dual of the linear operator $\psi^{2}$, so that $\left\langle s_{\lambda}, \psi^{2}\left(s_{\mu}\right)\right\rangle=\left\langle\phi^{2}\left(s_{\lambda}\right), s_{\mu}\right\rangle$ 
for all $\lambda, \mu \in \mathcal{P}$, it is a classical fact that $\phi^{2}\left(s_{\chi}\right)=\varepsilon_{2}(\chi) s_{\chi^{\prime}} s_{\chi^{\prime \prime}}$ when $\chi=\mathrm{cq}_{2}\left(\gamma, \chi^{\prime}, \chi^{\prime \prime}\right)$ for skew shapes $\chi^{\prime}, \chi^{\prime \prime}$ (see [Litw]; $\phi^{2}\left(s_{\chi}\right)=0$ if the shape $\chi$ admits no domino tableaux). Therefore by theorem 2.2.2:

$$
\phi^{2}\left(s_{\chi}\right)=\varepsilon_{2}(\chi) \sum_{\nu \in \mathcal{P}} \# \operatorname{Yam}_{2}(\chi, \nu) s_{\nu}
$$

(cf. [CaLe, Corollary 4.3, (5)]), whence the first member of (2) equals $\left\langle\phi^{2}\left(s_{\lambda}\right), s_{\mu}\right\rangle$. The second member is obtained by evaluating in two ways the sum of $(-1)^{\operatorname{Spin}(D)} x^{\mathrm{wt}(D)}$ as $D$ ranges over $\operatorname{SSDT}\left(\mu^{\square}, n\right)$. On one hand, since the map from domino tableaux to Yamanouchi domino tableaux in [CaLe, Theorem 7.3] preserves the spin, this sum decomposes as

$$
\sum_{\nu \in \mathcal{P}}\left(\sum_{\substack{M \in \operatorname{Yam}_{2}\left(\mu^{\square}, \nu\right) \\ T \in \operatorname{SST}(\nu, n)}}(-1)^{\operatorname{Spin}(M)} x^{\operatorname{wt}(T)}\right)=\sum_{\nu \in \mathcal{P}}\left(\sum_{M \in \operatorname{Yam}_{2}\left(\mu^{\square}, \nu\right)}(-1)^{\operatorname{Spin}(M)}\right) s_{\nu}(n) .
$$

On the other hand, one can cancel from the sum all contributions of domino tableaux $D$ among whose chains for $s_{1}$ (which are all closed) there is least one that can be moved (cf. [vLee3, proposition 4.3.1]): the tableau obtained by moving one such chain contributes with an opposite sign by [vLee3, proposition 4.4.1]. What remains are those domino tableaux $D \in \operatorname{SSDT}\left(\mu^{\square}, n\right)$ for which every $2 \times 2$ block corresponding to a square of $\mu$ is occupied by a pair of vertical dominoes with equal entries, forming a forbidden chain for $s_{1}$. These tableaux are in bijection with ordinary semistandard tableaux $D^{\prime}$ of shape $\mu$; $\operatorname{since} \operatorname{Spin}(D)=|\mu|$ and wt $D=2$ wt $D^{\prime}$, the summation becomes

$$
\sum_{D^{\prime} \in \operatorname{SST}(\mu, n)}(-1)^{|\mu|} x^{2 \mathrm{wt} D^{\prime}}=(-1)^{|\mu|} \psi^{2}\left(s_{\mu}\right)(n) .
$$

Taking the coefficient of $s_{\lambda}(n)$ in (4) and (5), one finds that $\left\langle s_{\lambda}, \psi^{2}\left(s_{\mu}\right)\right\rangle$ equals the second member of (2) (cf. [CaLe, Theorem 5.3]), which establishes that identity since $\left\langle s_{\lambda}, \psi^{2}\left(s_{\mu}\right)\right\rangle=\left\langle\phi^{2}\left(s_{\lambda}\right), s_{\mu}\right\rangle$.

Our combinatorial construction corresponding to (2) will consist of a bijection between tableaux $L \in \operatorname{Yam}_{2}(\lambda, \mu)$ and tableaux $M$ in a subset $B_{\lambda, \mu}$ of $\operatorname{Yam}_{2}\left(\mu^{\square}, \lambda\right)$, such that $L$ has half as many vertical dominoes as $M$ has horizontal dominoes (so that $\varepsilon_{2}(\lambda)=(-1)^{2} \operatorname{Spin}(L)=(-1)^{|\mu|-\operatorname{Spin}(M)}$ ), together with a proof that $\sum_{M \in C_{\lambda, \mu}}(-1)^{\operatorname{Spin}(M)}=0$, where $C_{\lambda, \mu}$ is the complement of $B_{\lambda, \mu}$ in $\operatorname{Yam}_{2}\left(\mu^{\square}, \lambda\right)$. That proof is similar to the argument leading to (5): we define $C_{\lambda, \mu}$ to be the subset of $\operatorname{Yam}_{2}\left(\mu^{\square}, \lambda\right)$ of tableaux that contain at least one chain for $s_{1}$ that can be moved while preserving the Yamanouchi property. The contributions of these tableaux to the sum will cancel out, provided we can show that the following holds:

2.3.1. Lemma. Let $M$ be a Yamanouchi domino tableau, $s \in\left\{s_{0}, s_{1}\right\}$, and let $S$ be the set of closed chains $C$ in $M$ for $s$, for which the tableau obtained from $M$ by moving $C$ is again a Yamanouchi domino tableau. Then the tableau obtained from $M$ by simultaneously moving the chains of any subset of $S$ is also a Yamanouchi domino tableau.

In other words, the set $S$ itself is not affected by moving any of its chains. This means that the relation between Yamanouchi domino tableaux of being obtainable from one another by moving a subset of the chains of $S$ is an equivalence relation, whose equivalence classes have size $2^{\# S}$; since moving any one chain changes the parity of $\operatorname{Spin}(M)$, the sum of $(-1)^{\operatorname{Spin}(M)}$ over such a class is 0 if $S \neq \emptyset$. A similar fact, but with "Yamanouchi" replaced by "semistandard", was needed in the derivation of (5), but that fact is simpler: it follows directly from [vLee3, proposition 4.3.1]. We note that a remark is made in [CaLe] (after its Lemma 8.5) that appears to claim the validity our lemma 2.3.1; however this remark is neither justified nor used there, and so we shall provide a proof of the lemma below.

Given $L \in \operatorname{Yam}_{2}(\lambda, \mu)$, we construct a filling $M$ of $Y\left(\mu^{\square}\right)$ using an augmentation of domino tableaux similar to that of ordinary tableaux in [vLee5, (15)], by attaching subscripts called ordinates to the entries 
of $L$. We do this in such a way that if the dominoes $d$ with fixed entry $L(d)=i$ are listed by decreasing value of $\operatorname{pos}(d)$ (i.e., from right to left), then their ordinates increase by unit steps, starting at 0 . This being done, the domino containing $i_{j}$ (entry $i$ with ordinate $j$ ) determines two dominoes of $M$, which occupy the $2 \times 2$ block $\{2 i, 2 i+1\} \times\{2 j, 2 j+1\}$ of squares in $Y\left(\mu^{\square}\right)$. These two dominoes will be horizontal if $d$ is vertical, and vice versa; their two entries are the row numbers of the two squares forming $d$ (which are equal if the dominoes are vertical, and different in they are horizontal, in which case of course the top domino gets the smaller entry). While it is not obvious that $M$ is a Yamanouchi (or even a semistandard) domino tableau, it is clear that it has twice as many horizontal dominoes as $T$ has vertical dominoes.

2.3.2. Theorem. For any $\lambda, \mu \in \mathcal{P}$ and $L \in \operatorname{Yam}_{2}(\lambda, \mu)$, the filling $M$ of $Y\left(\mu^{\square}\right)$ constructed above lies in the subset $B_{\lambda, \mu}$ of $\operatorname{Yam}_{2}\left(\mu^{\square}, \lambda\right)$ (i.e., none of its chains for $s_{1}$ can be moved without destroying the Yamanouchi property); moreover the construction defines a bijection $\operatorname{Yam}_{2}(\lambda, \mu) \rightarrow B_{\lambda, \mu}$.

As an example, consider $\lambda=(6,5,3,3,3)$ and $\mu=(4,3,2,1)$. Now there is just one $L \in \operatorname{Yam}_{2}(\lambda, \mu)$, displayed here together with the corresponding element $M \in \operatorname{Yam}_{2}\left(\mu^{\square}, \lambda\right)$, both with augmentation:
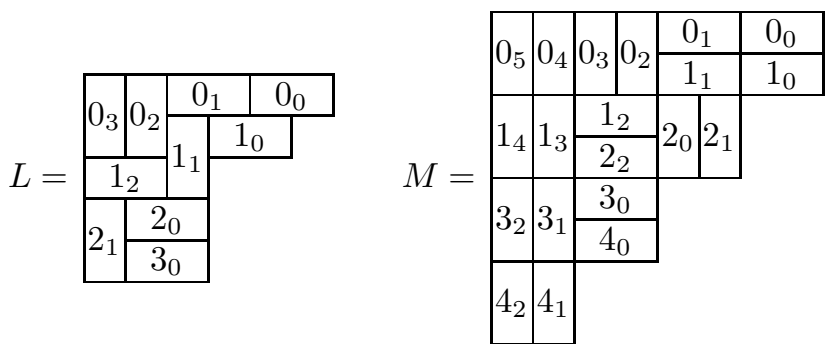

As can be seen, in addition to the fact that the entries of each pair of dominoes in a $2 \times 2$ block of $M$ give the row numbers of the squares of the corresponding domino of $L$, their ordinates give the column numbers. The four tableaux of the remainder $C_{\lambda, \mu}$ of $\operatorname{Yam}_{2}\left(\mu^{\square}, \lambda\right)$ form a single equivalence class, for which the set $S$ of lemma 2.3 .1 for $s_{1}$ consists of two closed chains, each occupying one $2 \times 2$ block:

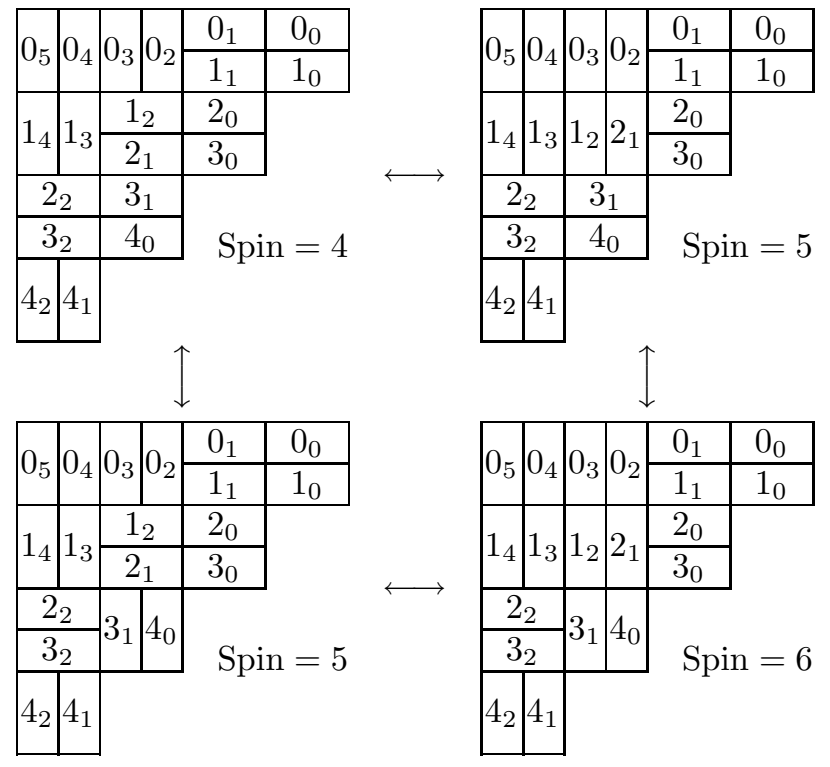

We have added ordinates in these tableaux as well; one can see that the blocks of the chains in $S$ have pairs $\left\{1_{2}, 2_{1}\right\}$ and $\left\{3_{1}, 4_{0}\right\}$ of entry-ordinate combinations that do not correspond to adjacent squares. 


\section{$\S 3$. Coplactic operations.}

We shall now study in detail the algorithm defining the bijection of [CaLe, Theorem 7.3], which is given in [CaLe, 7.1]. It is modelled after Robinson's algorithm, which is defined in [Rob, §5], but is more clearly described in [Macd, I 9]. The basic steps of Robinson's algorithm are the coplactic operations, for a the detailed discussion of which we refer to [vLee5]. Similar operations can be defined for domino tableaux as well; these are the basic steps of the first algorithm mentioned above, and will also be called coplactic operations. We recall that the basic definition of coplactic operations is the one for words, which is then transferred to ordinary semistandard tableaux by applying it to words obtained by reading the entries of the tableaux in some specific order. The transfer to semistandard domino tableaux is more involved, for two related reasons: there is no obvious way to apply the valid reading orders for ordinary tableaux to domino tableaux, and coplactic operations may involve rearrangement of dominoes (sometimes a coplactic operation is applicable, but no proper result can be obtained by just changing the entry of a domino).

The method of definition adopted in [CaLe] is to use a particular reading order called the column reading of a domino tableau, which detects whether a coplactic operation can be applied, and if so makes a change to one entry as a first approximation of the operation; in case the result is not a semistandard domino tableau, a transformation called $R_{1}$ or a sequence of transformations called $R_{2}$ is applied, yielding the desired result. This method is not without difficulties; the effect of the transformations on further coplactic operations is hard to perceive, as they entail permutations of the letters of the column reading. In particular it is not obvious that the "coplactic graph" associated to a domino tableau is always isomorphic to that of some ordinary tableau, or equivalently, of some word; in any case one cannot always take the column reading word. Yet this property is essential for establishing the bijection of [CaLe, Theorem 7.3], since the second component of the pair it associates to a domino tableau $D$ can be described as "the Young tableau that is in the same place within its coplactic graph as $D$ is" (because of a similar characterisation for Robinson's bijection, cf. [vLee5, §4.2]). Alternatively, our bijection $\pi_{1}$ could be taken as basis for the definition of coplactic operations on domino tableaux: it follows from [vLee5, theorem 3.3.1] that if $\pi_{1}(D)=(U, V)$, and a coplactic operation $c$ is applicable to $U$ (and $V$ ), then $(c(U), c(V)$ ) is again a self-switching tableau pair, whence one could define $c(D)=\pi_{1}^{-1}(c(U), c(V))$. Then the mentioned isomorphism of coplactic graphs is obvious, but it is not easy to understand directly the effect of a coplactic operation on a domino tableau. It is the essence of theorem 2.1.3 to establish a link between these two possible definitions of coplactic operations. As a preliminary step we shall in this section recast the definition of [CaLe] into a more manageable form that avoids the choice of a particular reading order.

\subsection{Augmented domino tableaux.}

As a basis of our definitions we shall use the notion of $\nu / \kappa$-dominance of domino tableaux, which refines that of Yamanouchi domino tableaux, and is analogous to the same notion for ordinary tableaux defined in [vLee5, 1.4.1]. Definitions using "companion tableaux" or a reading of the entries would, while possible, be less natural in the case of domino tableaux; nevertheless a simple definition is possible, using augmentation of the domino tableaux with ordinates added to the entries, as in the construction of theorem 2.3.2.

3.1.1. Definition. An augmentation of a semistandard domino tableau $D \in \operatorname{SSDT}(\lambda / \mu)$ for a skew shape $\nu / \kappa$ is an assignment of ordinates to all $d \in \operatorname{Dom}(D)$, such that the conditions below are satisfied; if such an augmentation exists (which is then unique) $D$ will be called $\nu / \kappa$-dominant. The combination of the entry $i$ and ordinate $j$ of a domino $d$ will be written as $i_{j}$, and $\operatorname{pos}(d)$ will then be denoted by $\pi\left(i_{j}\right)$.

a. Each $i_{j}$ occurs at most for one domino, and it does so if and only if $(i, j) \in Y(\nu / \kappa)$;

b. $\pi\left(i_{j+1}\right)<\pi\left(i_{j}\right)$ whenever $\{(i, j),(i, j+1)\} \subseteq Y(\nu / \kappa)$;

c. $\pi\left((i+1)_{j}\right)<\pi\left(i_{j}\right)$ whenever $\{(i, j),(i+1, j)\} \subseteq Y(\nu / \kappa)$.

Conditions a and $\mathrm{b}$ imply that among the dominoes with a fixed entry $i$, the ordinates increase from right to left by units steps, starting at $\kappa_{i}$; the test for $\nu / \kappa$-dominance then amounts to verification of condition c. Like for ordinary tableaux, $D$ can only be $\nu / \kappa$-dominant if wt $D=\nu-\kappa$, and we shall say that $D$ is simply $\kappa$-dominant if it is $\nu / \kappa$-dominant for $\nu=\kappa+$ wt $D$; note that it is still required that $\nu$ be a partition. We shall show that $D$ is a Yamanouchi domino tableau if and only if it is 0-dominant. 
The definition above appears to be symmetric with respect to entries $i$ and ordinates $j$, but is not really so due to the requirement that $D$ be a semistandard domino tableau: ordering dominoes by increasing entries and then (for equal entries) by decreasing ordinates defines a standard domino tableau. We shall see that it is possible as well to order dominoes by ordinate first; these should decrease, and among dominoes with equal ordinates, the entries should increase. In fact we shall prove the following.

3.1.2. Proposition. Let $D \in \operatorname{SSDT}(\lambda / \mu)$ be augmented for $\nu / \kappa$, and let ' $\prec$ ' be a total ordering of the set $\left\{i_{j} \mid(i, j) \in Y(\nu / \kappa)\right\}$ with the property that $i_{j} \preceq k_{l}$ whenever $i \leq k$ and $j \geq l$. Then there exists a standard domino tableau $D_{\prec}$, called the specialisation of $D$ for ' $\prec$ ', with $\operatorname{Dom}\left(D_{\prec}\right)=\operatorname{Dom}(D)$, and in which these dominoes are added in increasing order for ' $\prec$ ' of their entry-ordinate combinations in $D$.

This means essentially that a domino containing $i_{j}$ can be added to the tableau as soon as the dominoes containing $(i-1)_{j}$ and $i_{j+1}$ (if they exist) are present. The stronger formulation of the proposition is necessary however: requiring only $(i-1)_{j} \prec i_{j}$ and $i_{j+1} \prec i_{j}$ may fail to imply $i_{j} \prec k_{l}$ for $i<k$ and $j>l$ by transitivity, as intermediate entry-ordinate combinations may be absent (by contrast, definition 3.1.1 does imply $\pi\left(k_{l}\right) \leq \pi\left(i_{j}\right)$ whenever $k \geq i$ and $l \geq j$, by convexity of skew diagrams). Note that the orderings ' $\prec$ ' allowed by the proposition are just the valid reading orders of the skew diagram $Y(\nu / \kappa)$. To prove the proposition, we use the following general lemma (a standard fact of some theory, no doubt), which allows us to focus on changing the order between just a single pair of values.

3.1.3. Lemma. Let $X$ be a finite set, $S$ the set (of cardinal (\#X)!) of all total orderings of $X$, and $\Gamma$ the graph on $S$ in which there is an edge between two orderings if and only if they differ only in the comparison of a single pair of elements of $X$ (this is a Cayley graph of $\mathbf{S}_{\# X}$ ). Then for any partial ordering ' $<$ ' on $X$, the subgraph of $\Gamma$ on the orderings that extend ' $<$ ' is connected.

Proof. It is readily checked that any minimal length path in $\Gamma$ between points $s, t$ in the subgraph remains within the subgraph. Some may find the following form of this argument clearer: arranging $X$ in a linear array according to $s$, and then bubble sorting it using $t$ as sorting criterion, any comparable pair of elements of $X$ for ' $<$ ' remains in the correct relative order throughout the sorting process. $\square$

Proof of proposition 3.1.2. It will suffice to prove that if the proposition holds for some ' $\prec$ ', and $\left\{i_{j}, k_{l}\right\}$ with $i<k$ and $j<l$ are adjacent for ' $\prec$ ', then the proposition holds also for the ordering ' $\prec$ ' in which these two combinations are interchanged. To this end we consider the standard domino subtableau of $D_{\prec}$ consisting of the corresponding two dominoes. Using edge sequences one easily classifies the possible standard domino tableaux with the same shape as a given tableau with two dominoes $d, d^{\prime}$, in terms of $\left|\operatorname{pos}(d)-\operatorname{pos}\left(d^{\prime}\right)\right|$ : when this value is 2 , only one such tableau exists, when it is 1 , there are two tableaux, but with different sets of dominoes (in this case the skew diagram is a $2 \times 2$ block), and in the remaining case of a value exceeding $2\left(\operatorname{since} \operatorname{pos}(d)=\operatorname{pos}\left(d^{\prime}\right)\right.$ is impossible), there are two tableaux, with the same sets of dominoes, which are added in either order. In view of definition 3.1.1, we therefore only have to exclude the possibility that $\pi\left((i+1)_{j+1}\right)=\pi\left(i_{j}\right)-2$ for some $i, j$. Assuming that, one necessarily has $\pi\left(i_{j+1}\right)=\pi\left((i+1)_{j}\right)=\pi\left(i_{j}\right)-1$; calling this value $p$, the fact that the domino containing $i_{j+1}$ is followed in the standardisation of $D$, after some intermediate dominoes, by a domino containing $(i+1)_{j}$ that has the same position $p$, necessitates the presence among those intermediate dominoes of a domino with position $p+2$, and one with position $p-2$. Those intermediate dominoes are the ones with entry $i$ and ordinates $\leq j$, followed by those with entry $i+1$ and ordinates $>j$ (each ordered by decreasing ordinates); therefore the domino with position $p+2$ can only be the one containing $i_{j-1}$, while the one with position $p-2$ must be the one containing $(i+1)_{j+2}$. But then one has $\pi\left((i+1)_{j}\right)=\pi\left(i_{j-1}\right)-2$ and $\pi\left((i+1)_{j+2}\right)=\pi\left(i_{j+1}\right)-2$; taking $j$ to be either minimal or maximal, one arrives at a contradiction. $\square$

As an instance of this proposition, each augmented domino tableau has a specialisation for the Kanji order of entry-ordinate pairs $\left(i_{j} \prec k_{l}\right.$ whenever $j>l$, or $j=l$ and $\left.i<k\right)$. Consequently, the union of a pair of dominoes $a, b$ respectively containing $i_{j}$ and $(i+1)_{j}$ is a skew diagram; in particular, $a$ does not extend to the left beyond $b$ (as in $\frac{a}{b}$ ), nor does $b$ extend to the right beyond $a$ (as in $\frac{a}{b}$ ).

3.1.4. Proposition. A semistandard domino tableau is Yamanouchi if and only if it is 0-dominant. 
Proof. Recall that a domino tableau is Yamanouchi when its column reading is a Yamanouchi word, and that the column reading orders dominoes by their leftmost column, and in case of equality by their bottommost row. Yamanouchi words can be characterised as follows: if one attaches to each letter an ordinate counting the occurrences of the same letter to its right in the word, then for any letter $(i+1)_{j}$, the letter $i_{j}$ occurs to its right. It is easily verified that if $D$ is a Yamanouchi domino tableau, then attaching these ordinates of letters to the corresponding entries of $D$, the conditions of definition 3.1.1 are satisfied: one obtains an augmentation of $D$ for $\nu / 0$, where $\nu=$ wt $D$. Conversely, let $D$ be a semistandard domino tableau augmented for $\nu / 0$; if $(i+1)_{j}$ occurs, then so will $i_{j}$, and by the remarks above, the domino containing $(i+1)_{j}$ will precede the one containing $i_{j}$ in the column reading. $\square$

\subsection{Coplactic operations on domino tableaux.}

We now proceed to define coplactic operations on domino tableaux, using augmentations. A similar description of coplactic operations for ordinary tableaux can be found in [vLee5, §3.4]; it essentially consists of performing a jeu de taquin slide on the set of entry-ordinate pairs, viewed as coordinates of squares. In the case of domino tableaux we proceed similarly, but a few additional cases have to be treated. We shall first describe this procedure, then show that this gives a proper definition of coplactic operations, and finally show that the operations defined in [CaLe] produce the same effect as ours.

Like in the case of ordinary tableaux, the coplactic operations $e_{i}$ and $f_{i}$ only involve the part of the tableau with entries $i$ and $i+1$; for the purpose of defining these operations, we may therefore ignore any other dominoes. So let $D$ be a semistandard domino tableau, all of whose entries are in $\{i, i+1\}$, and for the moment consider only the operation $e_{i}$. There exists some $\kappa$ for which $D$ is $\kappa$-dominant (it suffices that $\kappa_{i}-\kappa_{i+1}$ be sufficiently large), and we equip $D$ with the augmentation for $\nu / \kappa$, where $\nu=\kappa+$ wt $D$. If $\kappa_{i}=\kappa_{i+1}$, then $e_{i}$ cannot be applied ( $D$ is then 0-dominant, hence Yamanouchi); otherwise we shall construct a $\kappa^{\prime}$-dominant tableau $E$ with corresponding augmentation, where $\kappa^{\prime}$ is obtained from $\kappa$ by decreasing the part $\kappa_{i}$ by 1 . It may be that $D$ is already is $\kappa^{\prime}$-dominant, in which case we shall get $E=D$ (only ordinates will have changed); if so, the construction is restarted with the new augmentation found (in other words, $\kappa$ is replaced by $\kappa^{\prime}$ ). The more interesting case is when $D$ is not $\kappa^{\prime}$-dominant (so $\kappa_{i}-\kappa_{i+1}$ is minimal for $\kappa$-dominance of $D$ ); then obviously $E \neq D$, and we define $e_{i}(D)=E$.

We construct $E$ (with its augmentation) by transforming the augmentation of $D$ in a sequence of steps, which as indicated above resemble those of jeu de taquin, performed on the set of entry-ordinate combinations. At each stage there is one such combination $i_{j}$ with $(i, j) \in Y\left(\nu / \kappa^{\prime}\right)$ that does not occur. Then we consider the combinations $i_{j+1}$ and $(i+1)_{j}$; if neither occurs (so $(i, j)$ is a corner of $Y(\nu)$ ), then the process terminates. Otherwise, the process may proceed by changing one of these combinations into $i_{j}$ (written $i_{j} \leftarrow i_{j+1}$ or $i_{j} \leftarrow(i+1)_{j}$ ): this simple case applies when either just one of these two combinations occurs, or when the union of the dominoes containing these combinations forms a skew diagram; the change then affects the domino $d$ of these two for which $\operatorname{pos}(d)$ is larger.

In the remaining case that dominoes exist containing $i_{j+1}$ and $(i+1)_{j}$, but their union is not a skew diagram, one of the following two transformations is applied, which rearrange several dominoes at once. To allow a compact display, we subtract $i$ from all entries and $j$ from all ordinates, so that for instance $i_{j+1}$ and $(i+1)_{j}$ are drawn respectively as $0_{1}$ and $1_{0}$. The first possible transformation is

$$
\mathrm{T}_{0}: \quad \mathrm{C}_{0}=\begin{array}{|l|l|}
\hline 0_{1} \\
\hline 1_{1} & 1_{0} \\
\hline
\end{array} \quad \longrightarrow \quad \begin{array}{c|c|c|}
\hline 0_{1} & 0_{0} \\
\hline 1_{0} \\
\hline
\end{array} .
$$

The second possible transformation may involve an arbitrarily large number of dominoes; the largest part of $D$ that matches the left hand pattern $\mathrm{C}_{1}$ is transformed into the corresponding right hand pattern:

$$
\mathrm{T}_{1}: \quad \mathrm{C}_{1}=\begin{array}{|l|l|l|l|l}
\hline 0_{k} & \ldots & 0_{2} & 0_{1} & \\
\hline 1_{k} & \ldots & 1_{2} & 1_{1} & \\
\hline
\end{array} \quad \rightarrow \quad \begin{array}{|l|l|l|l|l|}
0_{k} & 0_{k-1} & \ldots & 0_{1} & 0_{0} \\
\hline 1_{k-1} & \ldots & 1_{1} & 1_{0} \\
\hline
\end{array} .
$$

We define the active region of the application of $e_{i}$ in the simple case as the domino whose entry changes, and otherwise as the pattern $\mathrm{C}_{0}$ or $\mathrm{C}_{1}$ being transformed. 
As an example, consider the following domino tableau $D$, augmented for $\lambda / \mu=(5,5,4) /(2,1,0)$.

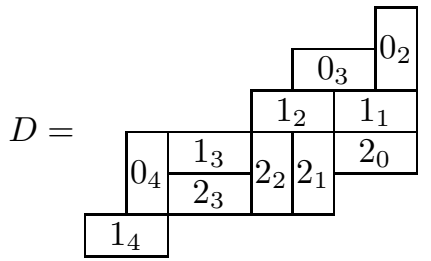

Application of $e_{0}$ is achieved by the sequence of replacements $0_{1} \leftarrow 0_{2} \leftarrow 0_{3} \leftarrow 1_{3} \leftarrow 1_{4}$ (where we ignore the dominoes with entry 2); these only involve the simple case of the definition, since each of the pairs of dominoes containing $\left\{0_{2}, 1_{1}\right\},\left\{0_{3}, 1_{2}\right\}$, and $\left\{0_{4}, 1_{3}\right\}$ occupies a skew diagram. Application of $e_{1}$ to $D$ however involves the replacement $1_{0} \leftarrow 1_{1}$, followed by a transformation $\mathrm{T}_{0}$ changing the configuration

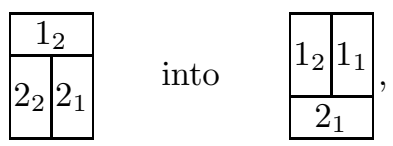

and finally the replacement $2_{2} \leftarrow 2_{3}$; one therefore finds

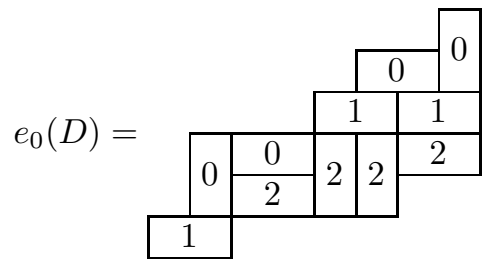

and

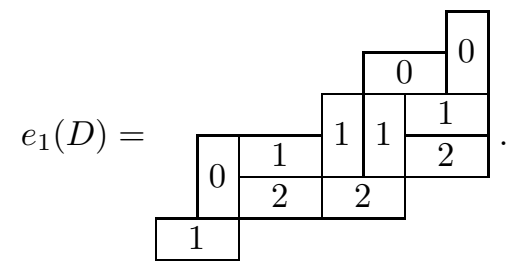

Note that retaining the ordinates found after computing $e_{0}(D)$, one would not get an augmentation of that tableau, which is a consequence of ignoring the entries 2 in the construction, in order to isolate an application of $e_{0}$ : while it would in a way be more natural to end the process with $1_{3} \leftarrow 2_{3}$ instead of $1_{3} \leftarrow 1_{4}$, that would compute $e_{1}\left(e_{0}(D)\right)$ in one go rather than $e_{0}(D)$ (we shall say a bit more about this below). As we are in fact computing just $e_{i}(D)$, there is no real need to continue the process after the "hole" has propagated into row $i+1$ of $Y\left(\nu / \kappa^{\prime}\right)$, either after a replacement $i_{j} \leftarrow(i+1)_{j}$ or a transformation $\mathrm{T}_{0}$ or $\mathrm{T}_{1}$; this helps however to relate to the inverse operation $f_{i}$. That operation is defined by reversing the above process in the obvious ways: entry-ordinate pairs are modified as in an outward jeu de taquin slide, with in addition the inverse transformations of $\mathrm{T}_{0}$ and $\mathrm{T}_{1}$. Applying $f_{i}$ to $e_{i}(D)$ constitutes a step-by-step reversal of the application of $e_{i}$ to $D$.

Observe in the computation of $e_{1}(D)$ that the augmented tableau contains $\frac{13}{23} 2_{2}$, matching $\mathrm{C}_{1}$ for $i_{j}=1_{2}$ and $k=1$; nevertheless $\mathrm{T}_{1}$ does not apply, since the "hole" never reaches $1_{2}$ (there is no replacement $1_{1} \leftarrow 1_{2}$ because the dominoes containing $\left\{1_{2}, 2_{1}\right\}$ in $D$ do not form a skew diagram).

We shall now proceed to show that the definition above is a proper one, i.e., that it handles all cases that can arise, and that the result is always a semistandard domino tableau. Assume first that the simple case of the definition applies at all steps; then the result can be seen to be semistandard by comparison of dominoes, as follows. To facilitate the formulation, let us define relations ' $<_{\mathrm{h}}$ ' and ' $<_{\mathrm{v}}$ ' between dominoes, where $a<_{\mathrm{h}} b$ and $a<_{\mathrm{v}} b$ both imply that $\operatorname{pos}(a)<\operatorname{pos}(b)$ and that $a \cup b$ is a skew diagram, in addition to which $a<_{\mathrm{h}} b$ means that there is a standard domino tableau that adds the domino $a$ and then $b$, and $a<_{\mathrm{v}} b$ that there is a standard domino tableau that adds $b$ and then $a$. Then either $a<_{\mathrm{v}} b<_{\mathrm{h}} c$ or $a<_{\mathrm{h}} b<_{\mathrm{v}} c$ imply $\operatorname{pos}(c)-\operatorname{pos}(a) \geq 3$, and hence both $a<_{\mathrm{h}} c$ and $a<_{\mathrm{v}} c$. We may assume there is a replacement of the form $i_{j} \leftarrow(i+1)_{j}$ (otherwise there is nothing to prove); denote the domino affected by that replacement by $d$, and by $d_{k, l}$ any other domino containing $k_{l}$ at that moment. Now from the original contents of the dominoes, and the tests leading to the replacement $i_{j} \leftarrow(i+1)_{j}$, one deduces 
$d_{i+1, j+1}<_{\mathrm{v}} d_{i, j+1}<_{\mathrm{h}} d<_{\mathrm{h}} d_{i+1, j-1}<_{\mathrm{v}} d_{i, j-1}$ (with the proviso that some of the $d_{k, l}$ may not exist, but the dominoes actually present form a contiguous subsequence); the required semistandardness follows.

In the case that at some point the dominoes containing $i_{j+1}$ and $(i+1)_{j}$ do not unite to a skew diagram, we argue using the same notation, again referring to the contents of dominoes at this point. By proposition 3.1.2, there is a standard domino tableau that adds the dominoes of $D$ in the following order of their original contents: $i_{j+1},(i+1)_{j+1}, i_{j},(i+1)_{j}$ (where $(i+1)_{j+1}$ and/or $i_{j}$ may be absent). The domino originally containing $i_{j}$, if present, is now $d_{i, j-1}$, and since $d_{i+1, j}<_{\mathrm{h}} d_{i+1, j-1}<_{\mathrm{v}} d_{i, j-1}$, it can be interchanged in the ordering with $d_{i+1, j}$; this leaves $d_{i+1, j+1}$ as the only domino possibly intervening between $d_{i, j+1}$ and $d_{i+1, j}$, and since by assumption $d_{i+1, j}$ cannot be added directly after $d_{i, j+1}$, the domino $d_{i+1, j+1}$ must be present. It then follows that one either has a configuration $\mathrm{C}_{0}$, or $\mathrm{C}_{1}$ for $k=1$; after extending the latter if possible to a maximal match of $\mathrm{C}_{1}$, one finds the transformation $\mathrm{T}_{0}$ or $\mathrm{T}_{1}$ that is to be applied. The fact that the result of this transformation is semistandard is verified easily: for $\mathrm{T}_{0}$ one uses that presence of $(i+1)_{j+2}$ implies that of $i_{j+2}$; for $\mathrm{T}_{1}$, one uses that the presence of $(i+1)_{j+k+1}$ implies that of $i_{j+k+1}$, plus the maximality of the match of $\mathrm{C}_{1}$, and $d_{i+1, j}<_{\mathrm{h}} d_{i, j-1}$ as found above.

For tableaux with entries $i$ and $i+1$ only, the final entry-ordinate pairs define a proper augmentation, so $e_{i}(D)$ is $\kappa^{\prime}$-dominant, as claimed. To obtain a similar result in the presence of arbitrary entries, the construction simulating jeu de taquin can be extended across more rows of $Y\left(\nu / \kappa^{\prime}\right)$, as was hinted at above. This requires extending the configurations in (6) downwards, in a similar way as the configurations in (7) are extended leftwards, again choosing a maximal match for the transformation to be applied. The effect of this extended construction on the underlying domino tableau (forgetting the ordinates) will be application of a sequence $e_{i}, e_{i+1}, \ldots$ of operations with increasing index. If $D$ is $\kappa$-dominant and $\kappa^{\prime}$ is obtained from $\kappa$ by decreasing the part $\kappa_{i}$ by 1 , then the length of this sequence is the minimal one for which the final result is $\kappa^{\prime}$-dominant; this is the same as what happens in the case of ordinary tableaux [vLee5, theorem 3.4.1]. Since this construction will not be used in the sequel, we leave the precise formulations and verifications to the interested reader; they are quite similar to the ones above.

We now verify that the operations of [CaLe] coincide with ours; we may assume all entries to be in $\{i, i+1\}$. First observe that there can be no other dominoes sharing a column with the active region; therefore it splits the column reading word into a prefix, an active part, and a suffix. Then the prefix is a Yamanouchi word, and the suffix is an anti-Yamanouchi (i.e., reverse anti-dominant) word; to see this one reasons as in the proof of proposition 3.1.4, using the ordinates at the time of the replacement $i_{j} \leftarrow(i+1)_{j}$, or of the transition $\mathrm{T}_{0}$ or $\mathrm{T}_{1}$. For the simple case this already shows that application of $e_{i}$ to $D$ induces application of $e_{i}$ on the reverse column reading word; for the other cases it reduces the verification to that for $\mathrm{C}_{0}$ and $\mathrm{C}_{1}$. The definitions of [CaLe] produce

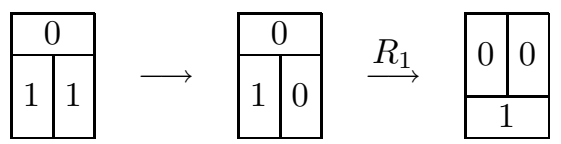

in the first case and

\begin{tabular}{|c|c|c|c|c|c|c|c|c|c|c|c|c|c|c|c|c|c|c|}
\hline 0 & $\ldots$ & 0 & 1 & 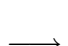 & 0 & . & 0 & 0 & $R_{2}$ & 0 & $\ldots$ & 0 & 0 & $R_{2} \ldots R_{2}$ & 0 & 0 & $\cdots$ & 0 \\
\hline 1 & $\ldots$ & 1 & 1 & $\longrightarrow$ & 1 & $\ldots$ & 1 & 10 & & 1 & $\ldots$ & 10 & 1 & & 0 & 1 & $\ldots$ & 1 \\
\hline
\end{tabular}

in the second case; in either case the overall effect matches that of $\mathrm{T}_{0}$ respectively $\mathrm{T}_{1}$. 


\section{$\S 4$. Self-switching tableau pairs and coplactic operations.}

In this section we shall prove that under the bijection $\pi_{1}$ of proposition 2.1.1, application of a coplactic operation to a semistandard domino tableau $D$ corresponds to application of the same operation to each component of the associated pair $(U, V)=\pi_{1}(D)$. This will immediately prove theorem 2.1.3: the second component in the bijection of [CaLe, Theorem 7.3] is constructed from the sequence of raising operations that transform $D$ into a Yamanouchi domino tableau, and since $U$ and $V$ have coplactic graphs isomorphic to that of $D$, the result is the same as the second component of Robinson's bijection applied to $U$ or $V$, which is the Young tableau associated to either of them by jeu de taquin (cf. [vLee5]). This commutation of $\pi_{1}$ with coplactic operations is easy to verify in the basic configurations affected by these operations, in the various situations that can arise. Nevertheless, the proof in the general case is difficult, because of the way $\pi_{1}$ is defined in terms of the standardisation of $D$, and the fact that in terms of the standardisation, the effect of coplactic operations is not localised (although it is in terms of dominoes and entries). The reduction to the case that $D$ contains entries $i$ and $i+1$ is fairly easy, but to further reduce to a situation simple enough to allow a uniform description of the entire computation of $\pi_{1}(D)$ presents difficulty, as relatively distant dominoes intervene in the standardisation. We shall resolve this problem by showing that other specialisations of an augmentation of $D$ can also be used to compute $\pi_{1}(D)$, in particular the one for the Kanji order; the augmentation will be the one used in defining the coplactic operation.

\subsection{Self-switching tableau pairs and augmentation.}

In order to show that alternative orders of dominoes can be used to compute $\pi_{1}(D)$, let us consider conditions under which the interchange of two successive entries in a standard domino tableau leads under application of $\pi_{0}$ to the interchange of the corresponding entries in each component of the pair of self-switching standard tableaux. Interchanging the order of two successive entries in a standard domino or ordinary tableau (when this is possible) will affect exactly one partition in the associated chain; the case we are interested in is when in the tableau switching family computing $\pi_{0}$, the change of the member $\lambda^{[k, k]}$ causes a change of the members $\lambda^{[k, j]}$ and $\lambda^{[i, k]}$ for all $i, j$, and of no others. Now if in a tableau switching family $\left(\lambda^{[i, j]}\right)_{i \in I, j \in J}$ with $I=\{k-1, k, k+1\}$, none of the skew diagrams $Y\left(\lambda^{[k+1, j]} / \lambda^{[k-1, j]}\right)$ is a domino, then another tableau switching family can be obtained by changing each partition $\lambda^{[k, j]}$ into the unique other partition between $\lambda^{[k+1, j]}$ and $\lambda^{[k+1, j]}$. This amounts to observing that if during some sequence of slides applied to a skew tableau of size 2, the two squares never become adjacent, then the same sequence of slides would arise if the entries of the two squares were interchanged (since apparently the entries are never being compared); a formal proof can also easily be given by analysing all possible cases for $J=\{l, l+1\}$. In order that our tableau switching family for $\pi_{0}$ satisfy the given description, it is necessary that the subfamily with $i \in\{k-1, k, k+1\}$ be of this type (and its "transpose" subfamily with $j \in\{k-1, k, k+1\}$ will then be similar), and that it is still of this type when its members $\lambda^{[k-1, k]}, \lambda^{[k, k]}$, and $\lambda^{[k+1, k]}$ are changed (this addition means the dominoes $Y\left(\lambda^{[k, k]} / \lambda^{[k-1, k-1]}\right)$ and $Y\left(\lambda^{[k+1, k+1]} / \lambda^{[k, k]}\right)$ are non-adjacent, so that their entries can indeed be interchanged). Recall that for ordinary tableaux one has the notions of $\nu / \kappa$-dominance and augmentations, with the analogue of proposition 3.1.2; calling orderings ' $\prec$ ' described in that proposition "valid", we have the following.

4.1.1. Proposition. Let $D$ be a semistandard domino tableau, and $(U, V)=\pi_{1}(D)$. Then for any skew shape $\nu / \kappa$, each of $U$ and $V$ is $\nu / \kappa$-dominant if and only if $D$ is; if so, for any valid ordering ' $\prec$ ' of $Y(\nu / \kappa)$, the specialisations $D_{\prec}, U_{\prec}$, and $V_{\prec}$ of their augmentations for $\nu / \kappa$ are related by $\pi_{0}\left(D_{\prec}\right)=\left(U_{\prec}, V_{\prec}\right)$.

We shall derive the second part first. To be precise, we assume that $U$ and $V$ are $\nu / \kappa$-dominant, and attach ordinates to the dominoes of $D$ in such a way that conditions a and b of definition 3.1.1 are satisfied; we then show that there is a standard domino tableau $D_{\prec}$ in which the dominoes of $\operatorname{Dom}(D)$ are added in increasing order for ' $\prec$ ', and that $\pi_{0}\left(D_{\prec}\right)=\left(U_{\prec}, V_{\prec}\right)$. This is obvious when ' $\prec$ ' is the Semitic order (the specialisations are then standardisations), so by lemma 3.1.3, it suffices to prove that the stated property for a valid ordering ' $\prec$ ' implies the same for another such ordering ' $\prec$ ' that differs from it by the exchange of two consecutive entry-ordinate pairs for ' $\prec$ ', which are necessarily of the form $\left\{i_{j}, k_{l}\right\}$ with $i<k$ and $j<l$. Since jeu de taquin preserves $\nu / \kappa$-dominance and is compatible with 
taking specialisations (cf. [vLee2, theorem 5.1.1]), the squares containing $i_{j}$ and $k_{l}$ are not adjacent in any tableau obtained by jeu de taquin from $U_{\prec}$ (one has $\pi\left(i_{j}\right)<\pi\left(i_{l}\right)<\pi\left(k_{l}\right)$, where $\pi\left(x_{y}\right)=\operatorname{pos}(s)$ for the square $s$ containing $\left.x_{y}\right)$. It follows that the tableau switching family for the computation of $\pi_{0}\left(D_{\prec}\right)$ is of the type described above with respect to the interchange of $i_{j}$ and $k_{l}$, whence $\pi_{0}\left(D_{\prec^{\prime}}\right)=\left(U_{\prec^{\prime}}, V_{\prec^{\prime}}\right)$ as required.

It remains to prove the first part of the proposition. To show that in the argument above condition c of definition 3.1.1 is satisfied for the ordinates added to $D$, we apply what we have just proved for the case that ' $\prec$ ' is the Kanji order. Then any pairs $i_{j}$ and $(i+1)_{j}$ are consecutive, and by the argument used to prove proposition 2.1.1, the positions of the dominoes containing these pairs in $D$ are ordered in the same sense as positions of the corresponding squares of $U$, which gives the required condition. For proving conversely that $U$ and $V$ are $\nu / \kappa$-dominant whenever $D$ is, we cannot yet apply the relation $\pi_{0}\left(D_{\prec}\right)=\left(U_{\prec}, V_{\prec}\right)$, so a direct proof is needed, using standardisations to compute $(U, V)=\pi_{1}(D)$. To show that the conditions required for the candidate augmentations of $U$ and $V$ for $\nu / \kappa$ hold, we may separately verify them for each restriction to a subtableau with entries in $\{i, i+1\}$ only. Since such a restriction is obtained by jeu de taquin from a component of the tableau switching pair computed from the similar restriction of $D$, we may assume that $D$ itself has entries in $\{i, i+1\}$ only. Moreover, we may prove $\nu / \kappa$-dominance for any intermediate tableau in the tableau switching family considered (establishing $(U, V)=X(U, V))$, in place of $U$ and $V$; we choose the one at the transition between the slides into squares with entry $i$ and with entry $i+1$. For any $i_{j}$ and $(i+1)_{j}$, it follows from definition 3.1 .1 and proposition 3.1.2 that the outward square of the domino of $D$ containing $i_{j}$ has a position that is strictly larger than that of the inward square of the domino containing $(i+1)_{j}$; from these, the squares of the indicated intermediate tableau containing the same combinations are obtained by a sequence of outward respectively inward jeu de taquin slides, whence their positions are ordered in the same sense.

If we accept the validity of theorem 2.1.3, which will be established in the next subsection, the proposition allows us to generalise (3), or more precisely the equivalent identity $\left\langle\phi^{2}\left(s_{\chi}\right), s_{\nu}\right\rangle=\varepsilon_{2}(\chi) \# \operatorname{Yam}_{2}(\chi, \nu)$ :

4.1.2. Corollary. For all skew shapes $\chi, \psi$ one has

$$
\left\langle\phi^{2}\left(s_{\chi}\right), s_{\psi}\right\rangle=\varepsilon_{2}(\chi) D(\chi, \psi),
$$

where $D(\chi, \psi)$ is the number of $\psi$-dominant tableaux in $\operatorname{SSDT}(\chi)$.

Proof. If the bijection of [CaLe, Theorem 7.3] maps $D \in \operatorname{SSDT}(\chi)$ to $(Y, P)$ with $Y \in \operatorname{Yam}_{2}(\chi, \lambda)$ and $P \in \operatorname{SST}(\lambda)$, then it follows from proposition 4.1.1, theorem 2.1.3, and the fact that jeu de taquin preserves $\psi$-dominance, that $P$ is $\psi$-dominant if and only if $D$ is. Then $D(\chi, \psi)$ is equal to the sum, taken over all $\lambda \in \mathcal{P}$, of $\# \operatorname{Yam}_{2}(\chi, \lambda)$ times the number of $\psi$-dominant tableaux in $\operatorname{SST}(\lambda)$. The latter number equals $\left\langle s_{\lambda}, s_{\psi}\right\rangle$, so that we get $\varepsilon_{2}(\chi) D(\chi, \psi)=\sum_{\lambda \in \mathcal{P}}\left\langle\phi^{2}\left(s_{\chi}\right), s_{\lambda}\right\rangle\left\langle s_{\lambda}, s_{\psi}\right\rangle=\left\langle\phi^{2}\left(s_{\chi}\right), s_{\psi}\right\rangle$. $\square$

\subsection{Commutation of $\pi_{1}$ with coplactic operations.}

Let us state explicitly what we mentioned earlier, that coplactic operations can be applied to self-switching tableau pairs; this follows by applying [vLee5, theorem 3.3.1] twice to the identity $X(U, V)=(U, V)$.

4.2.1. Proposition. If $c$ is a coplactic operation and $(U, V)$ a self-switching tableau pair, then $c$ can be applied to $U$ if and only if it can be applied to $V$; if so, $(c(U), c(V))$ is a self-switching tableau pair.

This subsection is devoted to proving the following theorem; as we have observed above, it justifies the definition of coplactic operations for domino tableaux, and it implies theorem 2.1.3.

4.2.2. Theorem. If $c$ is a coplactic operation, $D$ a semistandard domino tableau, and $(U, V)=\pi(D)$, then $c$ can be applied to $U$ and $V$ if and only if it can be applied to $D$, and then $(c(U), c(V))=\pi_{1}(c(D))$.

The proof of this theorem will consist of a reduction in two steps to a situation sufficiently simple to be verified by an explicit computation. Both reduction steps are similar in nature, although the second one is much more subtle; they consist of dividing $D$ into three regions, of which only the middle one 
is affected by the coplactic operation, and showing that $U$ and $V$ have a similar division. In order to allow the desired conclusion to be drawn we need a small lemma, whose formulation is more awkward than that its claim is actually difficult. Recall that standard (domino) tableaux $S, T$, which are just chains of partitions, can be joined together into $S \mid T$ when their shape match, i.e., the last partition of $S$ coincides with the first one of $T$. We shall use a similar concatenation of semistandard tableaux, but do not insist that the result correspond to another semistandard tableau (although it will in the final applications of the construction); it is defined just as a formal symbol that need only determine a well defined standard tableau. Then we extend $\pi_{1}$ to an operation $\pi_{2}$ defined for such concatenations, using the fact that tableau switching essentially uses only the structure of standard tableaux. Formally we define $\pi_{2}\left(D_{0}|\cdots| D_{k}\right)=\left(U_{0,0}|\cdots| U_{0, k}, V_{0, k}|\cdots| V_{k, k}\right)$, where $U_{i, j}$ and $V_{i, j}$ are defined inductively for $0 \leq i \leq j \leq k$ by $\left(U_{i, i}, V_{i, i}\right)=\pi_{1}\left(D_{i}\right)$, followed by $\left(U_{i, j}, V_{i, j}\right)=X\left(V_{i, j-1}, U_{i+1, j}\right)$ for $i<j$. Then we have

4.2.3. Lemma. If $\pi_{2}\left(D_{0}\left|D_{1}\right| D_{2}\right)=\left(U_{0}\left|U_{1}\right| U_{2}, V_{0}\left|V_{1}\right| V_{2}\right)$, and if $D_{1}$ satisfies $\pi_{1}\left(c\left(D_{1}\right)\right)=(c(U), c(V))$ where $(U, V)=\pi_{1}\left(D_{1}\right)$, for a coplactic operation $c$, then $\pi_{2}\left(D_{0}\left|c\left(D_{1}\right)\right| D_{2}\right)=\left(U_{0}\left|c\left(U_{1}\right)\right| U_{2}, V_{0}\left|c\left(V_{1}\right)\right| V_{2}\right)$.

Proof. This follows immediately from the definition, using [vLee5, theorem 3.3.1] where appropriate.

This lemma directly allows the first reduction, which is to the case that $D$ has entries in $\{i, i+1\}$ only, when $c=e_{i}$ or $c=f_{i}$. The subtableaux $D_{0}, D_{1}$, and $D_{2}$ of $D$ are those whose dominoes have entries $<i$, in $\{i, i+1\}$, and $>i+1$ respectively; then $\left(U_{0}\left|U_{1}\right| U_{2}, V_{0}\left|V_{1}\right| V_{2}\right)$ corresponds to $(U, V)=\pi_{1}(D)$, and clearly $c(U)$ and $c(V)$ respectively correspond to $U_{0}\left|c\left(U_{1}\right)\right| U_{2}$ and $V_{0}\left|c\left(V_{1}\right)\right| V_{2}$. Here a concatenation of semistandard tableaux is said to correspond to another semistandard tableau if their outer shapes are the same, and the set of dominoes with their entries of the latter is the (disjoint) union of those of the components of the concatenation. In this case the relation is particularly simple, since the concatenation of the standardisations form the standardisation of the combined tableau, but that will not be the case for the second reduction.

Assume now that $D$ has entries in $\{i, i+1\}$ only, and that $c=e_{i}$ (the case $c=f_{i}$ will follow by reversal). The computation of $e_{i}(D)$ is achieved by modifying an augmentation of $D$ into an augmentation of $e_{i}(D)$. We shall specify below values $k$ and $l$, which are such that the entry-ordinate combinations $i_{k}$ and $(i+1)_{l}$ lie on the path of this "slide" (i.e., each is the missing combination at some point during the construction); assume for the moment that they are given. Let $D_{0}, D_{1}$, and $D_{2}$ be the semistandard subtableaux of $D$ of those dominoes which, at the point in the construction where $i_{k}$ is absent, have ordinates $>l$, in $\{l, l-1, \ldots, k\}$, and $<k$ respectively; similarly let $E_{0}, E_{1}$, and $E_{2}$ be the subtableaux of $e_{i}(D)$ of those dominoes whose ordinates satisfy the same conditions respectively, but at the point where $(i+1)_{l}$ is absent. Since any changes other than just changing ordinates take place between these two points in the construction, we have $E_{0}=D_{0}, E_{1}=e_{i}\left(D_{1}\right)$, and $E_{2}=D_{2}$, these are indeed subtableaux by proposition 3.1.2, and $D_{0}\left|D_{1}\right| D_{2}$ and $E_{0}\left|E_{1}\right| E_{2}$ respectively correspond to $D$ and to $e_{i}(D)$.

We can now apply lemma 4.2.3, but in order for the result to be of any use, we must ensure that concatenations like $U_{0}\left|U_{1}\right| U_{2}$ correspond to the proper semistandard tableau (in the mentioned case to $U$ in $(U, V)=\pi_{1}(D)$ ); this is where the specific choices of $k$ and $l$ become essential (for badly chosen values, $U_{0}\left|U_{1}\right| U_{2}$ might not even correspond to any semistandard tableau at all). The standard domino tableau $S$ associated to $D_{0}\left|D_{1}\right| D_{2}$ is far from being the standardisation of $D$; however, proposition 4.1.1 allows any specialisation of the augmentation of $D$ to be used to compute (the corresponding specialisations of) $U$ and $V$. Now $C$ is almost such a specialisation, but not quite; the only wrinkle is that, since one must of course use the original augmentation of $D$ in which the ordinates of all entries $i$ of $D_{2}$ are one more than at the point where $i_{k}$ is absent, the domino $d_{2}$ of $D_{2}$ initially containing $i_{k}$ (if present) is added in $C$ after the domino $d_{1}$ of $D_{1}$ containing $(i+1)_{k}$, whereas $i_{k} \prec(i+1)_{k}$ in any valid order ' $\prec$ '. We may assume that $i_{k}$ is present (otherwise we are already done), which implies the occurrence of $(i+1)_{k-1}$ (since $i_{k}$ changes into $\left.i_{k-1}\right)$; if the tableau switching family computing $(S, T)=\pi_{0}(C)$ can be shown to satisfy the conditions mentioned before proposition 4.1.1, with respect to interchange of $d_{1}$ and $d_{2}$, then $\pi_{2}\left(D_{0}\left|D_{1}\right| D_{2}\right)$ will correspond to $\pi_{1}(D)$. Similar considerations will be needed for $E_{0}\left|E_{1}\right| E_{2}$ and $e_{i}(D)$; here the dominoes containing $i_{l}$ and $(i+1)_{l}$ in the augmentation of $e_{i}(D)$ must be exchanged.

We start with considerations independent of the choice of $k$. Since the domino $d_{2}$ can be added 
after $d_{1}$, the two are not adjacent. We shall now argue that in the tableau switching family computing $\pi_{0}(C)$, the conditions required to the right of the diagonal member $\lambda^{[m, m]}$ affected by interchange of $d_{1}$ and $d_{2}$ are always met; these conditions are that $Y\left(\lambda^{[m+1, n]} / \lambda^{[m-1, n]}\right)$ is a not domino for any $n \geq m$. If any of them were, it would be a horizontal domino, since $\operatorname{pos}\left(d_{1}\right)<\operatorname{pos}\left(d_{2}\right)$. We claim that this cannot happen, because the square $s$ of the final difference $\lambda^{[m+1, n]} / \lambda^{[m, n]}$ (i.e., for maximal $n$ ) lies in a row strictly above that of $\lambda^{[m, m]} / \lambda^{[m-1, m]}$ (the outer square of $\left.d_{1}\right)$; since the squares of the supposed horizontal domino are obtained from these by inward respectively outward slides, this gives a contradiction. In fact, we show that $s$ lies strictly above the outer square $t$ of the domino $d_{3}$ of $D$ containing $(i+1)_{k-1}$, which lies weakly above the outer square of $d_{1}$. We augment $D_{2}$ as at its definition, so that $d_{2}$ contains $i_{k-1}$ and $d_{3}$ contains $(i+1)_{k-1}$; by proposition 4.1.1, the second component of $\pi_{1}\left(D_{2}\right)$ has a similar augmentation. Its square containing $i_{k-1}$, which is $s$, then lies strictly above the one containing $(i+1)_{k-1}$ which, being obtained by an outward slide from $t$, already at the bottom of its column in $D$, lies in the same row as $t$.

It remains to show that the conditions required to the left of $\lambda^{[m, m]}$ are also met. For this we need an additional assumption, namely that the square of $\lambda^{[m+1, m]} / \lambda^{[m, m]}$ (the inner square of $d_{2}$ ) lies strictly above any square of $D_{1}$; it then lies in particular above any square obtained by inward slides from that of $\lambda^{[m, m]} / \lambda^{[m-1, m]}$, which suffices as above. To verify the assumption, we choose $k$ to be the largest ordinate for which the inner square of the domino containing $i_{k}$ in the initial augmentation of $D$ lies in a row strictly above the active region for the application of $e_{i}$, or if no such $k$ exists, the one for which the combination $i_{k}$ is initially absent. The considerations for $e_{i}(D)$ are symmetric, and lead to choosing for $l$ the smallest ordinate for which the outer square of the domino containing $(i+1)_{l}$ in the final augmentation of $e_{i}(D)$ lies in a row strictly below the active region, or if no such $l$ exists, the one for which the combination $(i+1)_{l}$ is finally absent. With these choices, we have achieved a reduction of the verification of theorem 4.2.2 for $D$ to that for $D_{1}$.

Proof of theorem 4.2.2. By the above reductions we may assume that $D$ has entries in $\{i, i+1\}$ only, and that all dominoes lie in the rows containing the active region; we may moreover assume that if transforming the augmentation of $D$ into that of $e_{i}(D)$ starts with $i_{k}$ absent and ends with $(i+1)_{l}$ absent, then $k \leq j \leq l$ for all ordinates $j$. If the active region for the application of $e_{i}$ to $D$ consists either of a single domino or of a configuration $\mathrm{C}_{1}$, its top right corner contains $i+1$ in $D$, and its bottom left corner contains $i$ in $e_{i}(D)$, so that there can be no entries $i$ to the right nor entries $i+1$ to the left; it then follows that $D$ is reduced to the active region. In these cases the theorem is verified by a simple computation, displayed below. Like before we distinguish the components of the tableau switching pairs by italic and bold entries, and subtract $i$ from all entries, so that they become 0 or 1 ; we also display ordinates, running from 0 up to $r$, with $r^{\prime}=r-1$. Horizontal arrows denote applications of $e_{i}$ (to each component in case of tableau switching pairs), and vertical arrows applications of $\pi_{1}$ :

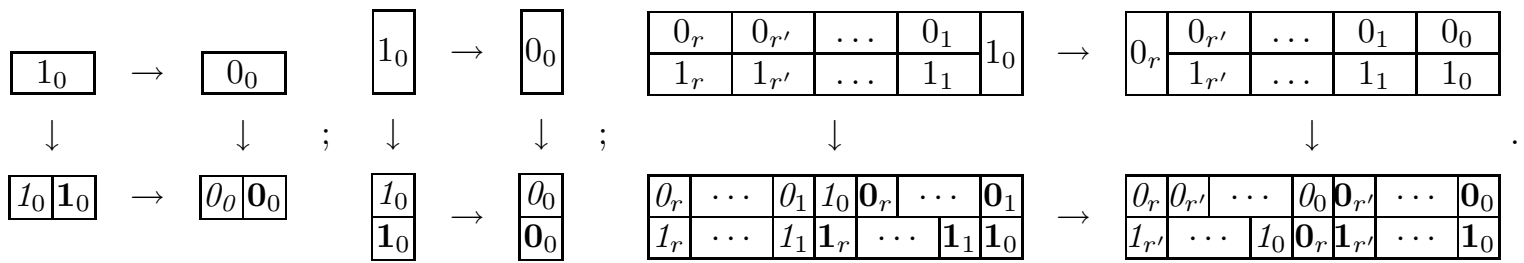

The case involving a transformation $\mathrm{T}_{0}$ is more complicated. In the top row of the active region, there may be horizontal dominoes with entry $i$ to its right, and in the bottom row there may be dominoes with entry $i+1$ to its left; these are accompanied by an equal number of entries $i+1$ respectively $i$ in the remaining rows, each of which may be divided into a number of horizontal and vertical dominoes. Hence this case is governed by four free parameters; in the diagram below these are determined by the values of the ordinates $0 \leq n<p \leq q \leq r$ (as before we subtract a common offset from all ordinates to make the smallest one equal to 0 ). We indicate certain sequences of horizontally repeated dominoes or squares with equal entries by an ellipsis, but to avoid excessive width, we alternatively indicate such repetition by displaying just one domino or square, with an asterisk attached to its entry; in that case the ordinate given is the leftmost (largest) one of the sequence. Primes attached to ordinates signify subtraction of 1. 


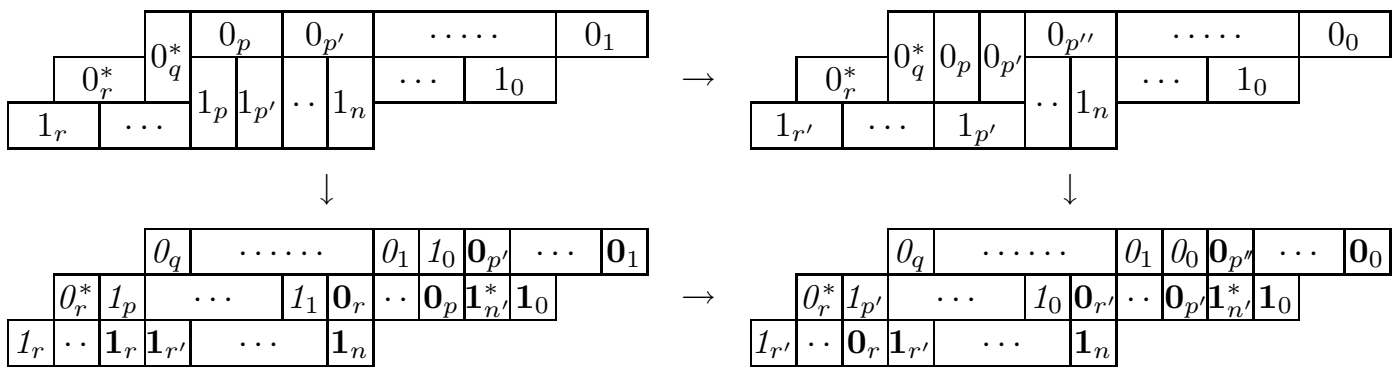

The shape of the tableaux is indicated only schematically, and away from the active region, vertical alignment is mostly accidental; however, in the bottom left diagram the alignment of $1_{p}$ and $\mathbf{1}_{r}$ and that of $1_{0}$ and $\mathbf{0}_{p}$ are always as indicated, as are the corresponding alignments in the bottom right diagram. It may be verified that the diagram correctly describes the computation for all possible parameter values.

\section{$\S 5$. Moving chains and coplactic operations.}

In this section we shall consider the same kind of questions as in the previous one, but replacing the correspondence $\pi_{1}$ by the operation of moving chains. In will be necessary to limit the type of chains in order to obtain the desired properties; in particular commutation with coplactic operations is obtained for the operation of moving open chains.

\subsection{Moving chains in augmented domino tableaux.}

Let a semistandard domino tableau $D$ be given, and a chain $C$ for $s \in\left\{s_{0}, s_{1}\right\}$ in $D$ that is not a forbidden chain, then [vLee3, proposition 4.3.1] tells us that it can be moved in $D$, resulting in another semistandard domino tableau $D^{\prime}$. We suppose that $D$ is $\nu / \kappa$-dominant, and ask under which circumstances the same is true for $D^{\prime}$; this generalises the question when moving a chain preserves the Yamanouchi property. It is easy to see that if we augment $D$ for $\nu / \kappa$, and move the ordinates of the dominoes in $C$ along with that domino, then conditions a and b of definition 3.1.1 are satisfied (in fact the forbidden chains are precisely those that would violate condition b); therefore our question is equivalent to asking whether condition $\mathrm{c}$ is satisfied. Fix $i, j$ for which there are dominoes in $\operatorname{Dom}(D)$ containing respectively $i_{j}$ and $(i+1)_{j}$, at least one of which lies in $C$. The positions of their fixed squares for $s$ are congruent modulo 2 , and a violation of condition c can only occur if these positions are equal. If so, we have a configuration

$$
\begin{array}{|c|}
\hline i_{j} \\
\hline i^{+}{ }_{j}
\end{array}
$$

(where $i^{+}$denotes $i+1$ ), with the fixed squares for $s$ on its main diagonal. If the top domino lies in $C$, then either the bottom domino is its successor in $C$, or there is another configuration (9) directly to the left of the one considered, but with ordinates $j+1$ instead of $j$; similarly if the bottom domino lies in $C$, then either the top domino is its successor in $C$, or there is another configuration (9), with ordinates $j-1$, to the right of this one. It follows that condition c of definition 3.1.1 is violated if and only if $C$ meets a configuration (9), in which case it is a closed chain, all of whose dominoes occur in such a configuration. The following definition covers both such pairs of dominoes and the ones forming a forbidden chain.

5.1.1. Definition. Let $D$ be an semistandard domino tableau, provided with an augmentation for $\nu / \kappa$. A pair of dominoes of $D$ is called blocked for $s \in\left\{s_{0}, s_{1}\right\}$ if the positions of their fixed squares for $s$ are equal, and their entry-ordinate combinations correspond to adjacent squares of $Y(\nu / \kappa)$. A chain in $D$ for $s$ is called blocked if any of its dominoes occurs in a blocked pair, and unblocked otherwise.

5.1.2. Proposition. Let $D$ be a semistandard domino tableau, provided with an augmentation for $\nu / \kappa$, and $C$ a chain in $D$ for $s \in\left\{s_{0}, s_{1}\right\}$. A $\nu / \kappa$-dominant tableau can be obtained from $D$ by moving $C$ if and only if $C$ is unblocked, which is the case as soon as any domino of $C$ is not part of a blocked pair. 
The notions of blocked pairs and blocked chains depend on the augmentation of $D$. However, open chains are never blocked, so in particular moving open chains in preserves the Yamanouchi property; this proves our second main theorem, 2.2.6. Also, whether or not a chain in an augmented tableau is blocked does not change when other (unblocked) chains are moved, which proves lemma 2.3.1.

5.1.3. Proposition. If $D$ is semistandard domino tableau, provided with an augmentation, then any unblocked chain $C$ in $D$ for $s$ is also a chain in any specialisation of the augmentation of $D$.

Proof. Let $D^{\prime}$ be the result of moving $C$ in $D$, which by proposition 5.1 .2 can be augmented for the same skew shape as $D$ was; for every occurring $i_{j}$, the dominoes of $D$ and $D^{\prime}$ containing that combination have their fixed square for $s$ in common. Then for any valid order ' $\prec$ ' the fact that $D_{\prec}$ and $D_{\prec}^{\prime}$ are standard domino tableaux (proposition 3.1.2) implies that $C$ is a chain of $D_{\prec}$. We may also formulate this as follows. To determine the successor and predecessor relations of the chain $C$, the entries in the standardisation of $D$ of certain pairs of dominoes in $\operatorname{Dom}(D)$ had to be compared, which is equivalent to comparing their entry-ordinate combinations using the Semitic order. The corresponding two dominoes in $\operatorname{Dom}\left(D^{\prime}\right)$ are always adjacent, and since $D_{\prec}^{\prime}$ is a well defined standard domino tableau, this implies that the comparison of their entry-ordinate combinations using ' $\prec$ ' gives the same result as using the Semitic order.

The fact that, by definition 5.1.1, the entry-ordinate combinations in a blocked pair of a domino tableau augmented for $\nu / \kappa$ define a domino inside $Y(\nu / \kappa)$, is the key to the construction of theorem 2.3.2.

Proof of theorem 2.3.2. Recall that, for each individual domino $d$ of an augmented Yamanouchi domino tableau in $\operatorname{Yam}_{2}(\lambda, \mu)$, we constructed a pair of dominoes forming a $2 \times 2$ block inside the diagram $Y\left(\mu^{\square}\right)$, with top-left corner at $(2 i, 2 j)$ if $d$ contains $i_{j}$. These dominoes, considered in isolation, can be made into a blocked pair for $s_{1}$, by giving them entry-ordinate combinations corresponding to the coordinates of the squares of $d$ (using the inward square of $d$ for the domino with the larger position). Note that these entries agree with the ones we assigned in the construction, and that the assignment of ordinates was also already shown in our example in $\S 2$. It is easy to see that this gives is a bijective correspondence between on one hand tilings $L$ of $Y(\lambda)$ with dominoes, each provided with an entry and ordinate, satisfying only condition a of definition 3.1.1 for $\nu / \kappa=\mu / 0$ (without requirement of belonging to a semistandard domino tableau), and on the other hand tilings $M$ of $Y\left(\mu^{\square}\right)$ with blocked pairs of dominoes, with the entries and ordinates again satisfying only condition a of definition 3.1.1, but for $\nu / \kappa=\lambda / 0$. What remains to be shown is that $L$ defines an augmentation of a semistandard domino tableau if and only if $M$ does so.

First note the semistandardness of an assignment of entries to a tiling of a diagram with dominoes can be tested by comparing adjacent dominoes: when two dominoes touch along a vertical edge, their entries should increase weakly from left to right, and when they touch along a horizontal edge, their entries should increase strictly from top to bottom. This test can be seen to succeed for $L$ or $M$ if the other satisfies definition 3.1.1. For $M$, we need only consider edges between different $2 \times 2$ blocks. For horizontal edges, the condition follows because both squares of the domino containing $(i+1)_{j}$ in $L$ lie strictly below those of the domino containing $i_{j}$. For vertical edges one similarly uses weak increase of row numbers from $i_{j}$ to $i_{j+1}$, which fails only in one case when $i_{j}$ and $i_{j+1}$ themselves fill a $2 \times 2$ block in $L$, but that case is readily checked to cause no problem either. Semistandardness of $L$ is verified similarly, but here row numbers are divided by 2 (and truncated to an integer), which causes strict increase down columns to be the subtler of the two conditions: if square $(r, c)$ belongs to a different domino of $L$ than $(r+1, c)$, then the domino of $M$ containing $r_{c}$ cannot lie entirely in an even row $2 i$, which forces its row number to remain strictly smaller than that of the domino containing $(r+1)_{c}$, even after division by 2 and truncation. The verification of conditions $\mathrm{b}$ and $\mathrm{c}$ of definition 3.1.1 proceed almost by reversal of these arguments, but in addition to monotonicity of entries one uses strict decrease of ordinates along rows and weak decrease down columns (which is easily checked), so that the difference $j-i$ for combinations $i_{j}$ decreases strictly both along rows and down columns (not counting the places where one stays within a domino, of course). For $M$ this suffices to establish the mentioned conditions, since the necessary comparisons of the positions of dominoes can be done based only on the $2 \times 2$ blocks containing them (which may be assumed distinct); for $L$ one observes that for these comparisons one may compare the contents of the inward dominoes of the corresponding 
$2 \times 2$ blocks in $M$, or equivalently the contents associated to the top-left squares of these blocks.

The fact that $L$ and $M$ have partition shapes plays no rôle at all in the proof above; these conditions are simply remnants of the fact that we had not yet introduced the notion of $\nu / \kappa$-dominance when stating theorem 2.3.2, but only the notion of Yamanouchi domino tableau. We can therefore generalise as follows:

5.1.4. Corollary. For any pair of skew shapes $\chi, \psi$ with $|\chi|=2|\psi|$, there is a bijection between the set of $\psi$-dominant domino tableaux $L$ of shape $\chi$, and the set of $\chi$-dominant domino tableaux $M$ of shape $\psi^{\square}$ for which there is no chain for $s_{1}$ that can be moved without destroying its $\chi$-dominance. The bijection is given by the construction of theorem 2.3.2, applied to the appropriate augmentations; in particular $2 \operatorname{Spin}(L)=|\psi|-\operatorname{Spin}(M)$ (i.e., $M$ has twice as many horizontal dominoes as $L$ has vertical dominoes).

In the particular case that $\chi=\lambda / \mu$ is a horizontal strip (its columns have length at most 1 ), every domino tableau $M$ of weight $\lambda-\mu$ is $\chi$-dominant. Then any $M$ in the corollary consists only of forbidden chains for $s_{1}$, and the set of all such $M$ is in bijection, not just with the indicated set of domino tableaux $L$, but more directly (by shrinking each forbidden chain to a single square) with the set of $M^{\prime} \in \operatorname{SST}(\psi)$ with wt $M^{\prime}=\frac{1}{2}(\lambda-\mu)$; it is the latter correspondence that was used in the derivation of equation (5).

\subsection{Coplactic operations and moving open chains.}

We have now proved all the facts announced in $\S 2$, but one more question deserves to be considered, namely whether coplactic operations commute with moving open chains. We already know that moving open chains does not affect the applicability of coplactic operations (since it preserves $\nu / \kappa$-dominance), and the close analogy to [vLee5, theorem 3.3.1] is a strong indication for expecting commutation. That one does in fact have commutation, as we shall now demonstrate, is therefore not surprising, but a proof still requires a considerable amount of work, and the verification of quite a few particular cases.

5.2.1. Theorem. Coplactic operations on domino tableaux commute with moving of open chains, in the following sense. If $D \in \operatorname{SSDT}(\chi), C$ is an open chain in $D$ for $s \in\left\{s_{0}, s_{1}\right\}$, and $D^{\prime}$ is obtained by moving $C$ in $D$, then $c(D)$ is defined if and only if $c\left(D^{\prime}\right)$ is, for any coplactic operation $c$; if so, the result of moving the open chain $C^{\prime}$ of $c(D)$ starting in the same square as $C$ is equal to $c\left(D^{\prime}\right)$.

Proof. We may assume that $c=e_{i}$, and (since the statement about applicability of coplactic operations is already proved) that $e_{i}(D)$ is defined. We set $E=e_{i}(D)$, and denote the result of moving $C^{\prime}$ in $E$ by $E^{\prime}$; we then have to show that $E^{\prime}=e_{i}\left(D^{\prime}\right)$. We provide $D$ with the augmentation that is transformed into one of $E$ by the definition of coplactic operations, and provide $D^{\prime}, E^{\prime}$, and $e_{i}\left(D^{\prime}\right)$ with corresponding augmentations.

Assume first that $C=C^{\prime}$ (i.e., these chains consist of the same sequence of dominoes; their entries and ordinates in $D$ respectively $E$ may differ); we must show that in this case the steps performed in the application of $e_{i}$ to $D$ (replacements of entry-ordinate pairs and possibly a transformation $\mathrm{T}_{0}$ or $\mathrm{T}_{1}$ ) are matched exactly in the application of $e_{i}$ to $D^{\prime}$. It is clear that if a transformation $\mathrm{T}_{0}$ or $\mathrm{T}_{1}$ occurs, the affected configuration $\mathrm{C}_{0}$ or $\mathrm{C}_{1}$ is disjoint from $C$, since all dominoes of $C$ must remain in $E$ in order to have $C=C^{\prime}$. It therefore suffices to show that every replacement $i_{j} \leftarrow i_{j+1}$ or $i_{j} \leftarrow(i+1)_{j}$ during the computation of $e_{i}(D)$ has its counterpart in the computation of $e_{i}\left(D^{\prime}\right)$. We may assume that both candidate combinations $i_{j+1},(i+1)_{j}$ for this replacement are present in the augmentation of $D$ (and of $D^{\prime}$ ), for otherwise the statement is obvious for lack of choice; let $\{x, y\} \subseteq \operatorname{Dom}(D)$ be the dominoes containing these combinations, with the one of $x$ actually being replaced (so $\operatorname{pos}(x)>\operatorname{pos}(y)$ ). Denoting by $x^{\prime}, y^{\prime} \in \operatorname{Dom}\left(D^{\prime}\right)$ the corresponding dominoes after moving $C$, it follows from $C^{\prime}=C$ that $x^{\prime}, y^{\prime} \in \operatorname{Dom}\left(E^{\prime}\right)$, and that $x^{\prime}$ contains $i_{j}$ in the augmentation of $E^{\prime}$, while $y^{\prime}$ contains the same combination $\left((i+1)_{j}\right.$ or $\left.i_{j+1}\right)$ as it does in the augmentation of $D^{\prime}$. This implies that the union of $x^{\prime}$ and $y^{\prime}$ is a skew diagram, and that $\operatorname{pos}\left(x^{\prime}\right)>\operatorname{pos}\left(y^{\prime}\right)$, which proves the required statement.

The remainder of the proof, which shows that in all cases with $C \neq C^{\prime}$ one has commutation as well, will appeal mainly to the kind of people who like to study the solutions of chess problems (but those who prefer to find the solution themselves may stop reading and do just that). We shall start by listing a number of variants in which the theorem can be seen to hold, and then show that with $C \neq C^{\prime}$, 
there is no way to avoid running into one of them. From any variant $\phi$, three others can be obtained by the following symmetries related to those of the "rectangle" of tableaux $\left[\begin{array}{cc}D & E \\ D^{\prime} & E^{\prime}\end{array}\right]$; these will not be listed separately. A variant denoted by $\phi^{\uparrow}$ is obtained by interchanging $D \leftrightarrow D^{\prime}$ and $E \leftrightarrow E^{\prime}$ and reversing the movement of the chains $C$ and $C^{\prime}$. A variant denoted by $\phi^{\leftrightarrow}$ is obtained by interchanging $D \leftrightarrow E$ and $D^{\prime} \leftrightarrow E^{\prime}$ while rotating all tableaux a half-turn and renumbering entries and ordinates in the opposite order, so as to interchange the rôles of $i$ and $i+1$. The third variant is $\phi^{\times}=\phi^{\uparrow \leftrightarrow}=\phi^{\leftrightarrow}$.

We do not make the assumption that $D$ has entries in $\{i, i+1\}$ only, since suppression of the remaining entries might change the structure of the chain $C$. However, we shall focus on a specific part of $D$ only, in which all entries are in $\{i, i+1\}$, and which in all cases has the property that the dominoes not lying in the explicitly indicated part of $C$ are member of a blocked pair for $s$, whence they cannot be part of $C$, regardless of the suppressed context (the situation for $E, D^{\prime}$ and $E^{\prime}$ is similar). Some cases involve transformations $\mathrm{T}_{1}$, and correspondingly have one or more parameters describing how often certain elements of the configurations are repeated horizontally. We include the case of zero repetitions, whenever this makes sense; we then also consider a replacement $i_{j} \leftarrow(i+1)_{j}$ in a vertical domino as an instance of the transformation $\mathrm{T}_{1}$ with $k=0$. We display entries with their ordinates (as before subtracting offsets to make the smallest ones 0 ), and indicate the relevant part of the open chain in each tableau by arrows. The fact that the chains and the effect of $e_{i}$ are as displayed, implies that any repeatable element is included in the display as often as the context allows. We start giving variants that we shall call $\alpha(k, l)$, for parameters $k \geq l \geq 0$ (variant $\alpha(0,0)$ has $C=C^{\prime}$, and may be excluded).

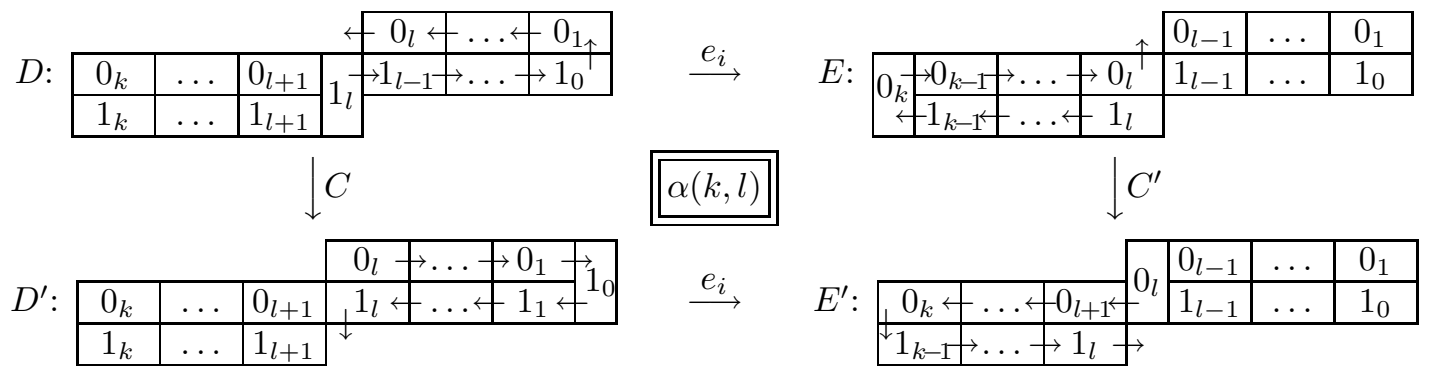

Note that variant $\alpha(k, l)^{\times}$coincides with $\alpha(k, k-l)$. Then one has variants $\beta(k)$ for $k>0$, obtained by modifying $\alpha(k, 0)$ slightly: upward arrows at the end of chains $C$ and $C^{\prime}$ in $\alpha(k, 0)$ are replaced by rightward arrows in $\beta(k)$, which does not affect the essential characteristics of the configuration.

\begin{tabular}{|c|c|c|c|c|c|}
\hline $0_{k}$ & $\ldots$ & $0_{1}$ & \multirow{2}{*}{$\overrightarrow{1_{0}}$} & \multirow{2}{*}{$\stackrel{e_{i}}{\longrightarrow}$} & \begin{tabular}{|l|l|l|l|}
$0_{k}$ & $\rightarrow 0_{k-1}$ & $\ldots \rightarrow 0_{0}$ \\
\end{tabular} \\
\hline $1_{k}$ & $\ldots$ & $1_{1}$ & & & \begin{tabular}{|c|c|c|}
$41_{k-1}$ & $\ldots+$ & $1_{0}$ \\
\end{tabular} \\
\hline & & \multicolumn{2}{|c|}{$C$} & $\beta(k)$ & $C^{\prime}$ \\
\hline $0_{k}$ & $\ldots$ & $0_{1}$ & $1_{0}$ & \multirow[t]{2}{*}{$e_{i}$} & \begin{tabular}{|c|c|c|c|}
$0_{k}$ & $+\ldots 0_{1}$ \\
\end{tabular} \\
\hline $1_{k}$ & $\ldots$ & $1_{1}$ & & & ${ }_{1} 1_{k-1} \rightarrow \ldots \rightarrow 1_{0} \rightarrow$ \\
\hline
\end{tabular}

A third family of variants is $\gamma(k)$ for $k \geq 1$. Here both a transformation $\mathrm{T}_{1}$ (for $e_{i}$ applied to $D$ ) and a transformation $\mathrm{T}_{0}$ (for $e_{i}$ applied to $D^{\prime}$ ) occur. Similar variants $\gamma^{\prime}(k)$ are obtained from $\gamma(k)$ by changing the direction at the beginning of the chains $C$ and $C^{\prime}$.

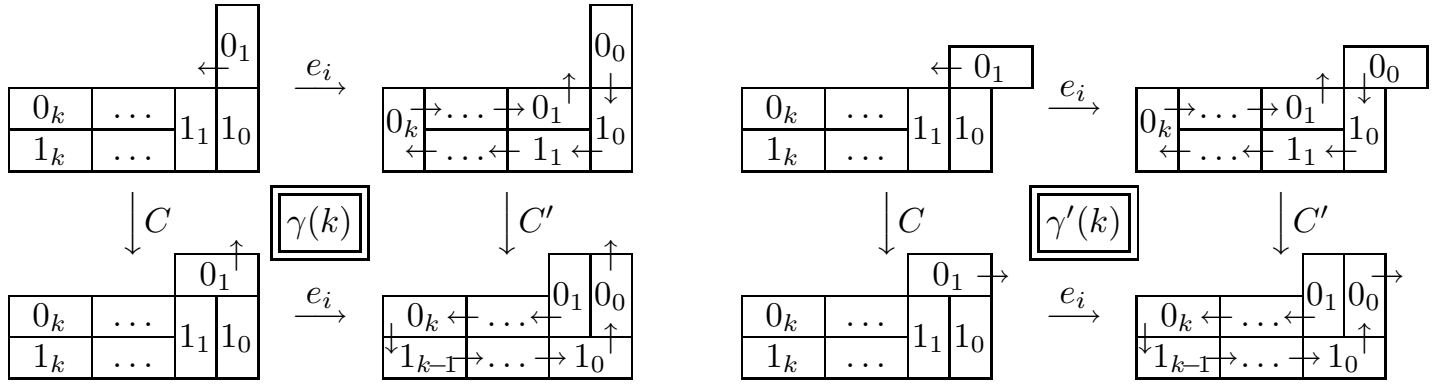


The final variants involve transformations $\mathrm{T}_{0}$ in applying $e_{i}$ both to $D$ and to $D^{\prime}$, but at different (overlapping) places; they have no parameters. The direction of $C$ and $C^{\prime}$ can be varied at both ends, giving 4 such variants: $\delta, \delta^{\prime}$ and $\delta^{\prime \prime}$ displayed below, and $\delta^{\prime \times}$.
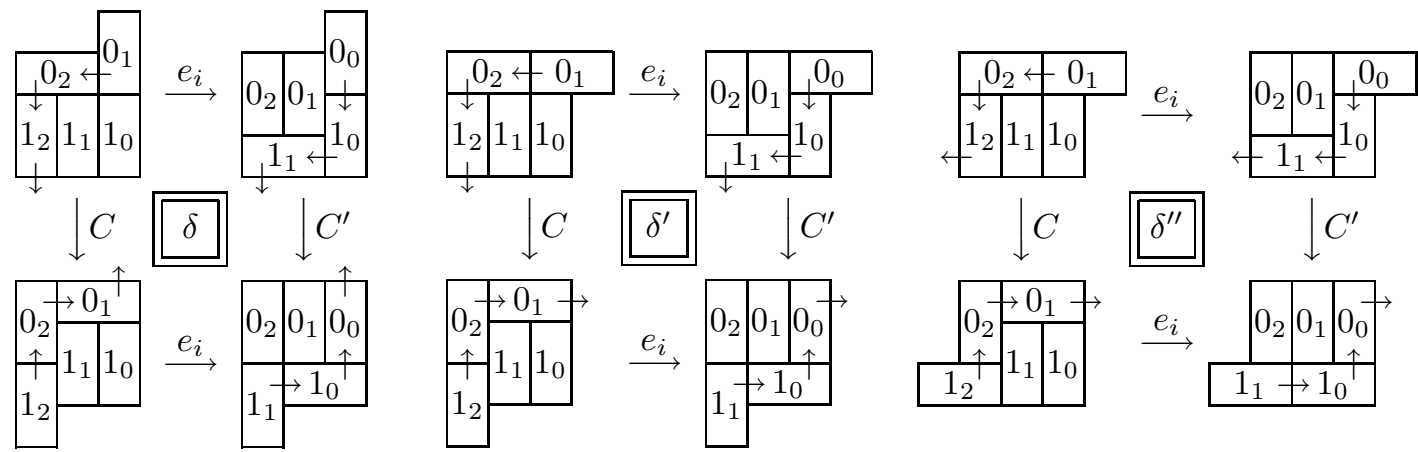

We now proceed to show that $C^{\prime} \neq C$ implies that, up to the mentioned symmetries, one of these variants arises. We label the cases that can arise in the application of $e_{i}$ to $D$ as follows: case $a$ means a replacement $i \leftarrow(i+1)_{j}$ occurs in a horizontal domino; case $b$ means that a transformation $\mathrm{T}_{0}$ occurs; case $c$ means that a transformation $\mathrm{T}_{1}$ occurs (which we take to include a replacement $i \leftarrow(i+1)_{j}$ in a vertical domino). In all cases the active region for the application of $e_{i}$ is rectangular, and of its top-left and bottom-right corner squares exactly one is fixed for $s$; by applying the symmetry $\phi \rightarrow \phi \leftrightarrow$ if necessary, we assume that it is the top left square.

Assuming $C \neq C^{\prime}$ as we do, there are two possibilities for the first point of divergence between $C$ and $C^{\prime}$ : (1) some domino of $C$ does not occur in $\operatorname{Dom}(E)$ (due to rearrangement of dominoes), and (2) the successor $y$ in $C$ of some domino $x$ does occur in $\operatorname{Dom}(E)$, but it is not the successor of $x$ in $C^{\prime}$. Recall from [vLee3, 4.1] that successor of $x$ is determined by comparing its entry to that of the domino occupying its "discriminant square", which is one step in the anti-diagonal direction away from the fixed square (for $s$ ) of $x$ (if the discriminant square lies outside $D$, then the test depends only on the shape of $D$, and we cannot have possibility (2)). Therefore, possibility (2) requires that the entry either of $x$ itself or of the domino containing the discriminant square is changed by $e_{i}$, and in such a way that the result of the comparison changes. Now in each of the cases there is a unique fixed square $t_{0}$ for $s$ for which application of $e_{i}$ changes the entry of its domino: $t_{0}$ is the left square of the active domino in case $a$, in case $b$ it is the right square of the middle row of $\mathrm{C}_{0}$, and in case $c$, it is the top-right corner of $\mathrm{C}_{1}$. Let $t_{0}$ be part of a domino $x_{0} \in \operatorname{Dom}(D)$ containing $(i+1)_{j}$, and let $t_{1}$ be the discriminant square of $x_{0}$ (it lies to the bottom-left of $t_{0}$ in case $a$, and to the top-right of $t_{0}$ otherwise); if in $D$ the square $t_{1}$ is part of a domino containing either $(i+1)_{j+1}$ (case $\left.a\right)$ or $i_{j}$ (other cases), then we define $x_{1}$ to be that domino. If possibility (2) applies, the crucial comparison must be between the entries of $x_{0}$ and $x_{1}$; moreover, in case $c$ it must be that $x=x_{1}$ (since $x_{0} \notin \operatorname{Dom}(E)$ ), but in case $b$, even if $x_{1}$ is defined, its successor lies in $\mathrm{C}_{0}$ and therefore not in $\operatorname{Dom}(E)$, which excludes possibility (2) altogether.

Suppose first that we are in case $a$; then $\operatorname{Dom}(D)=\operatorname{Dom}(E)$, so we have possibility (2). Since $x_{0}$ undergoes a replacement $i_{j} \leftarrow(i+1)_{j}$ and $x_{1}$ contains $(i+1)_{j+1}$ in $D$, there is a domino $x_{2}$ containing $i_{j+1}$ in $D$, with $x_{1}<_{\mathrm{v}} x_{2}<_{\mathrm{h}} x_{0}$. Since $x_{0}$ is horizontal, the only possible configuration is $\frac{x_{2}}{x_{1}} x_{0}$, so $x_{1}$ and $x_{2}$ form a blocked pair for $s$, which forces $x=x_{0}$. By including any further blocked pairs for $s$ with entries $i$ and $i+1$ occurring directly to the left of $x_{1}$ and $x_{2}$, we find an occurrence of $\beta(k)^{\downarrow}$.

Suppose next that we are in case $b$. Now the bottom left domino of $\mathrm{C}_{0}$ (containing $\left.(i+1)_{j+1}\right)$ is the successor for $s$ of the top domino (containing $i_{j+1}$ ). It may be that the remaining domino $x_{0}$ of $\mathrm{C}_{0}$ (containing $\left.(i+1)_{j}\right)$ forms a blocked pair for $s$ with a domino containing $(i+1)_{j-1}$ in $D$; if it does, $x_{1}$ is defined, and one gets one of the variants $\delta, \delta^{\prime}, \delta^{\prime \times}$, or $\delta^{\prime \prime}$. Otherwise $x_{1}$ may still be defined, but then since its contents is replaced $i_{j-1} \leftarrow i_{j}$ when applying $e_{i}$, it forms a $2 \times 2$ block of horizontal dominoes with a domino containing $(i+1)_{j-1}$ in $D$. Reasoning similarly we may find more such $2 \times 2$ blocks with entries $i$ and $i+1$ directly to the right, but the chain through $x_{0}$ eventually turns back and passes through the top and bottom left domino of $\mathrm{C}_{0}$; if $k$ blocks were found, we have a variant $\gamma(k+1)^{\times}$or $\gamma^{\prime}(k+1)^{\times}$. 
Suppose finally that we are in case $c$. If $x_{1}$ is not defined we have possibility (1), and the domino where $C$ and $C^{\prime}$ diverge must be $x_{0}$ (being in $\mathrm{C}_{1}$ and not part of a blocked pair); this leads to a variant $\alpha(k, 0)$ or $\beta(k)$. Otherwise, since $e_{i}$ causes a replacement $i_{j-1} \leftarrow i_{j}$ in $x_{1}$, there is a domino $x_{2}$ containing $(i+1)_{j-1}$ in $D$, with $x_{0}<_{\mathrm{h}} x_{2}<_{\mathrm{v}} x_{1}$, and it is the successor of $x_{0}$ for $s$ in $D$. If vertical, $x_{2}$ forms a blocked pair for $s$ with $x_{0}$, so that $C$ does not meet $\mathrm{C}_{1}$, and we must have possibility (2); this leads to variant $\gamma(k)$ or $\gamma^{\prime}(k)$. If $x_{2}$ is horizontal, then so is $x_{1}$, and the two form a $2 \times 2$ block. As above we may need to include more such blocks with entries $i$ and $i+1$ to the right, but the chain through $x_{0}$ eventually turns back to pass through $x_{1}$, excluding possibility (2); this leads to a variant $\alpha(k, l)$ with $l>0$.

For the record we mention that more grandiose versions of the commutation theorems 4.2.2 and 5.2.1 can be obtained, by replacing the coplactic operations by the extended construction simulating jeu de taquin on all entry-ordinate pairs that was indicated in subsection 3.2. The argument that reduces these commutation theorems to the ones given is the same as used for [vLee5, corollary 3.4.3]: the number of coplactic operations that correspond to a single jeu de taquin slide can be read off from the coplactic graph, and it therefore does not change when $\pi_{1}$ is applied or an open chain is moved. The theorems also have a consequence that does not refer to coplactic operations at all; we do not know a direct proof of it.

5.2.2. Corollary. If $D^{\prime}$ is obtained from $D$ by moving open chains, then $\pi(D)=\pi\left(D^{\prime}\right)$.

Proof. By theorems 5.2.1, 4.2.2, and [vLee5, theorem 3.3.1], we can reduce to the case that $D$ is a Yamanouchi domino tableaux, and $D^{\prime}$ will be one as well. But then $\pi(D)=\mathbf{1}_{\mathrm{wt} D}=\mathbf{1}_{\mathrm{wt} D^{\prime}}=\pi\left(D^{\prime}\right)$.

\section{$\S 6$. Concluding remarks.}

The complements we have given to the facts stated in [CaLe] result in a fairly complete and elegant theory for domino tableaux, which forms a nice parallel to the theory for ordinary tableaux, as described in [vLee5]. Yet, one may well wonder why all this works out so nicely, and if there is some deeper algebraic significance behind the combinatorial facts. The most general and natural algebraic interpretation of (augmented) domino tableaux that we know of, stated in corollary 4.1.2, seems to cry for a generalisation to higher plethysm operators, involving $r$-ribbon tableaux for $r>2$. However, none of the constructions of this paper generalise well to $r$-ribbon tableaux, and no reasonable generalisation of the definition of augmented (or even just Yamanouchi) domino tableaux seems to give the correct values (e.g., it is hard to decide which tableaux should correspond to $\left.\left\langle\phi^{3}\left(s_{(4,4,4)}\right), s_{(2,2)}\right\rangle=1=\left\langle\phi^{3}\left(s_{(3,3,3,3)}\right), s_{(2,2)}\right\rangle\right)$. The interested reader may check that every analysis in this paper depends on properties of domino tableaux that fail for $r$-ribbon tableaux with $r>2$ (or, as in the case of $\pi_{1}$, on constructions that have no analogue at all). One may surmise that the special place of domino tableaux has some relation with classical groups of types other than $A_{n}$, rather than just with plethysms; such a connection certainly does exist (see [Garf], where even moving chains play a rôle), but at present we cannot indicate any concrete links. 


\section{References.}

[BeSoSt] G. Benkart, F. Sottile, J. Stroomer, "Tableau switching: algorithms and applications", J. Combin. Theory, Ser. A 76, (1996), 11-43.

[CaLe] C. Carré and B. Leclerc, "Splitting the square of a Schur function into its symmetric and antisymmetric parts", J. of algebraic combinatorics 4, (1995), 201-231.

[FomSt] S. Fomin and D. Stanton, "Rim hook lattices", St. Petersburg Math. Journal 9, (1998), $1007-1016$.

[Garf] D. Garfinkle, "On the classification of primitive ideals for complex classical Lie Algebra's, II", Compositio Mathematica 81, (1992), 307-366.

[KaNa] M. Kashiwara and T. Nakashima, "Crystal graphs for representations of the $q$-analogue of classical Lie algebras", Journal of Algebra 165, (1994), 295-345.

[Knuth] D. E. Knuth, "Permutations, matrices and generalized Young tableaux", Pacific Journal of Math. 34, (1970), 709-727.

[LaSch] A. Lascoux and M. P. Schützenberger, "Le monoïde plaxique", Quad. Ricerca Scientifica C.N.R. 109, (1981), 129-156.

[LeTh] B. Leclerc and J.-Y. Thibon, "The Robinson-Schensted correspondence, crystal bases, and the quantum straightening at $q=0$ ", Electronic J. of Combinatorics 3 (no. 2), R11, (1996), 24 pp.

[vLee1] M. A. A. van Leeuwen, "The Robinson-Schensted and Schützenberger algorithms, an elementary approach", Electronic J. of Combinatorics 3 (no. 2), R15, (1996), 32 pp.

[vLee2] M. A. A. van Leeuwen, "Tableau algorithms defined naturally for pictures", Discrete Mathematics 157, (1996), 321-362.

[vLee3] M. A. A. van Leeuwen, "Edge sequcences, ribbon tableaux, and an action of affine permutations", European J. of Combinatorics 20, (1999), 179-195.

[vLee4] M. A. A. van Leeuwen, "An analogue of jeu de taquin for Littelmann's crystal paths", Séminaire Lotharingien de Combinatoire B41b, (1998), 23 pp.

[vLee5] M. A. A. van Leeuwen, The Littlewood-Richardson rule, and related combinatorics, math.CO/9908099, Submitted to Math. Soc. of Japan Memoirs.

[Litm1] P. Littelmann, "A Littlewood-Richardson rule for symmetrizable Kac-Moody algebras", Inventiones mathematicae 116, (1994), 329-346.

[Litm2] P. Littelmann, "A plactic algebra for semisimple Lie algebras", Advances in Math. 124 (2), (Dec. 1996), 312-331.

[Litw] D. E. Littlewood, "Modular representations of symmetric groups", Proc. Royal Soc. A 209, (1951), 333-353.

[Macd] I. G. Macdonald, Symmetric Functions and Hall Polynomials, Oxford Mathematical Monographs, Clarendon press, Oxford, 1979.

[Rob] G. de B. Robinson, "On the representations of the symmetric group", American Journal of Math. 60, (1938), 745-760.

[StWhi] D. W. Stanton and D. E. White, "A Schensted algorithm for rim hook tableaux", J. Combin. Theory, Ser. A 40, (1985), 211-247. 